\title{
Human EHMT2 activates p53 through methylation-independent mechanism
}

Miran Rada ${ }^{1}$, Elena Vasilieva ${ }^{2}$, Larissa Lezina ${ }^{1,2}$, Diana Marouco ${ }^{1}$, Alexey Antonov ${ }^{2,3}$, Sal Macip $^{1}$, Gerry Melino ${ }^{3}$ and Nickolai A Barleve,

${ }^{1}$ Department of Biochemistry, University of Leicester, Leicester LE1 9HN, UK;

${ }^{2}$ Institute of Cytology, Saint-Petersburg, 194064, Russia;

${ }^{3}$ MRC Toxicology Unit, Leicester, LE1 9HN, UK

*Correspondent author: nick.a.barlev@gmail.com

Running title: orthologues of G9a regulate p53 differentially

Keywords: G9a, p53, histone methyltransferase protein, histone acetyltransferase, CBP. 


\section{ABSTRACT}

p53 is a critical tumor suppressor in humans. It functions mostly as a transcriptional factor and its activity is regulated by numerous post-translational modifications. Among different covalent modifications found on p53 the most controversial one is lysine methylation. We found that human G9a (hG9a) unlike its mouse orthologue (mG9a) potently stimulated p53 transcriptional activity. Both ectopic and endogenous hG9a augmented p53-dependent transcription of pro-apoptotic genes, including Bax and PUMA, resulting in enhanced apoptosis and reduced colony formation. Significantly, siRNA-mediated knockdown of hG9a attenuated p53-dependent activation of PUMA. On the molecular level, hG9a interacted with histone acetyltransferase, p300/CBP, resulting in increased histone acetylation at the promoter of PUMA. The bioinformatics data substantiated our findings showing that positive correlation between G9a and p53 expression is associated with better survival of lung cancer patients. Collectively, this study demonstrates that depending on the cellular and organismal context, orthologous proteins may exert both overlapping and opposing functions. Furthermore, this finding has important ramifications on the use of G9a inhibitors in combination with genotoxic drugs to treat p53-positive tumors. 


\section{INTRODUCTION}

The p53 protein is a potent tumour suppressor that converts stress signals into cellular responses via activation or repression of many genes (Vousden \& Ryan 2009). Mutations in the TP53 genes have been reported in more than $50 \%$ of all types of cancers ${ }^{3}$. p53, known as a "guardian of genome", responses to various forms of cytotoxic stress by preventing the proliferation of genetically damaged cells through the control of their cell cycle and/or apoptosis. p53 executes its stress-response programmes through regulation of expression of its target genes, including p21, GADD45 $\alpha$ (cell cycle arrest) ${ }^{4,5}, \mathrm{PUMA}^{6}, \mathrm{BAX}^{7}, \mathrm{NOXA}^{8}, \mathrm{DR} 5$, p53AIP, PIG3, APAF1 (apoptosis) ${ }^{5}$, BTG2, and PaI1(senescence) ${ }^{5}$. In addition to proteincoding genes p53 also controls a number of micro-RNA and long non-coding genes (Hermeking H., p53 enters the microRNA world, Cancer Cell 2007; Lezina et al, CDDIS 2013; Zhang A., Xu M, Mo YY, Role of the lncRNA-p53 regulatory network in cancer, 2014 J Mol Cell Biol). In the unstressed cells p53 is a short-lived protein, which is rapidly degraded by ubiquitin-dependent mechanism in proteasomes (Pant V and Losano G Genes \& Dev. 2014. 28: 1739-1751; Grigoreva T et al, Oncotarget, 2015). Upon genotoxic stress, p53 becomes stabilized via multiple post-translational modificaitions (PTMs), including phosphorylation, acetylation, methylation to name just a few (Marouco et al, Oncotarget, 2015). Importantly, these genotoxic-induced PTMs also down-regulate the proteolytic activity of proteasomes (Moiseeva T et al, Oncotarget, 2013; Fedorova et al., 2011 BBRC) thus potentially further stabilizing p53. In the context of genotoxic stress-induced PTMs three major regulatory PTMdependent mechanisms that control p53 activity have been identified: subcellular localization of $\mathrm{p} 53^{2,12}$, its protein-protein interactions ${ }^{3,11-14}$, and finally, protein stability of the $\mathrm{p} 53$ protein itself $^{15-17}$.

Importantly, most of the lysine-specific PTMs that control activity and stability of p53 are carried out by histone-modifying enzymes (reviewed in Sims RJ Reinberg D 2008 Nat Rev 
Mol Cell Biol and ${ }^{1}$ ). For example, acetylation by histone acetyltransferase (HAT) p300/CBP renders p53 not only more stable, but also more transcriptionally active (Brooks CL Gu W 2011 Protein Cell; Espinosa JM and Emerson BM 2001 Mol Cell; Barlev et al, Mol Cell 2001). Methylation by lysine methyltransferase (KMT) Set7/9 augments the activity of p53 by promoting its acetylation via sequestering deacetylase (HDAC) Sirt1 (Ivanov et al, MCB 2007; Liu X Wang D PNAS 2011, Set7/9 regulates p53 activity by interacting with Sirtuin 1). Huang et al. (Huang et al, Nature, 2006) (2006) described another KMT, Smyd2, which acted, in contrast to Set7/9, as repressor of p53 activity. Additionally, Set8 and mouse G9a were also shown to inhibit p53 under non-stressed conditions (Shi X, Kachirskaia I et al 2007, Mol Cell; Huang J, Dorsey J et al, JBC 2010 G9a and Glp methylate lysine 373 in the tumor supressor $\left.\mathrm{p} 53^{27}\right)$.

G9a, a SET-domain histone methyltransferase protein, is expressed as multiple isoforms (Brown SE, Campbell RD, Sanderson CM Mamm Genome, 2001). The amino terminus of G9a comprises sequence of the NG36 gene, which was previously thought to be an independent gene. Recent study from the Batsche group (Mauger O et al, NAR 2015) suggests that G9a may be spliced differently depending on the tissue type. Interestingly, the alternatively spliced exon 10 in the G9a gene contains multiple phosphorylation sites, which may be important for signal transduction upon speicific stimulus.

G9a heterodimerises with G9a-like protein (GLP) via its carboxyl terminal SET domain to exert the full spectrum of its substrate methylation specificity ${ }^{18,19}$. Both proteins (independently and in the complex) catalyse mono- and dimethylation of lysine 9 and lysine 27 of $\mathrm{H} 3$ (H3K9 and H3K27, respectively) ${ }^{20-22}$ and histone $\mathrm{H} 1$ isotype 4 (H1.4) (Trojer et al. 2009; Weiss et al. 2010). In addition, G9a was reported to mono-methylate lysine 56 of histone H3 (H3K56) during G1 phase to regulate DNA replication (Yu Y, Grunstein M et al., 2012 Mol Cell). Furthermore, G9a and GLP methylate non-histone substrates, among them CEBP $\beta$ 
(K39), WIZ (K305), ACINUS (K654), G9a (automethylation), MyoD (K104) CDYL1 (K135), HDAC1, DNMT1, KLF12 ${ }^{25}$, and reptin (K67) ${ }^{21,26}$, and p53 (K373) ${ }^{27}$.

Notwithstanding the current consensus on the role of G9a in regulation of transcriptional activity as a transcriptional co-repressor, recent studies have challenged this view by showing that G9a also positively regulates gene expression and thus acts as a co-activator ${ }^{25,30-33}$. In this respect, G9a was recently reported as a transcriptional coactivator for Glucocorticoid Receptor (GR), by forming a molecular scaffold to recruit and facilitate binding of other co-activating proteins including GRIP1, CARM1 and p300 32,34. Interestingly, the underlying mechanism of G9a function as a positive regulator is methylation-independent, because UNC0646 (G9aspecific methyltransferase inhibitor) can not abolish this feature ${ }^{34},{ }^{30}$.

Taken together, these data suggest that G9a/GLP exerts pleiotropic functions in respect to transcriptional regulation, which may differ depending on the interaction with a particular transcription factor and/or chromatin environment of a particular gene.

Here, we report an unexpected finding that human and mouse G9a orthologues regulate p53 in opposite ways. Specifically, the human version of G9a (hG9a) augmented the activity of p53 towards its target genes irrespective of the methylation, while the mouse one (mG9a) blunted p53-dependent transcription in the methylation-specific manner. Futhermore, we uncovered the molecular mechanism of G9a-mediated activation of p53, which relied on the recruitment by G9a of a at least one coactivator HAT, CBP. The latter event resulted in increased histone acetylation and activation of the p53 target genes, including PUMA. 


\section{RESULTS}

\section{hG9a induces p53 transcriptional activity}

Several HMTs were shown to methylate p53 in the regulatory domain and thus regulate its activity (Fig. 1A) (Marouco et al, Oncotarget 2013). Since the carboxyl terminus of p53 is rich in lysines we decided to screen for additional HMTs that may methylate p53 and control its activity. A panel of recombinant GST-SET domains from twenty various HMTs (a gift from Dr Binda, Newcastle, UK) was analysed for methylation of p53 in vitro (the full list of HMTs tested is available upon request). Under these experimental conditions we could not detect any new HMTs, besides the already known ones, that robustly methylated p53 (data not shown). Surprisingly, in contrast to previously published data ${ }^{27}$ the GST-G9a SET fusion protein gave a very weak methylation signal (data not shown). We hypothesized that G9a may affect p53 activity in vivo. Thus, we examined the effect of human G9 and several other HMTs that might regulate p53 independently of its methylation. To this end, we chose Smyd3, a close relative of p53-specific HMT, Smyd2, and SetD2, which was shown previously to interact with p53 (Xie P et al., Histone methyltransferase protein SETD2 interacts with p53 and selectively regulates its downstream genes Cell Signal. 2008 Sep;20(9):1671-8). H1299 (p53 -/-) human lung carcinoma cells transfected with p53 and HTM-coding plasmids were tested in luciferase assay for the ability of HMTs to control the activation of p53-dependent Puma-luciferase reporter (Fig 1B). Smyd2 and Set7/9 were used as negative and positive controls, respectively. As expected, p53 alone activated transcription of the luciferase reporter over 15-fold compared to the pCDNA3 vector alone. Surprisingly, opposite to our expectations, expression of hG9a further augmented p53-dependent activation of the luciferase gene 1.8-fold (Fig. 1B). On the contrary, the reporter activity was reduced 1.5 -fold in the presence of Smyd2, Smyd3 and SetD2. Expectedly, Set7/9 displayed a modest but reproducible increase of p53-depedent activation of the Puma reporter (Chuikov et al, Nature 2004). The augmentation of p53 reporter 
activity by G9a was not due to overexpression of p53 since all the transfected samples contained comparable amounts of p53 as judged by western (Fig. 1B, middle panel).

To elucidate this unexpected result further we decided to compare side by side the effect of hG9a and its mouse orthologue on transcription of three bona fide p53 target genes: Bax, Puma, and p21 (Fig. 1D). The human G9a gene has two splice variants: hG9a long and hG9a short (Fig. 2C), which are also known as isoform-a and b. Isoform-a is a long isoform of G9a (hG9a(L)) composed of 1210 amino acids. This isoform involves two open reading frames: NG36 (amino acids 1-209) and G9a (amino acids 210-1210). Isoform-b is a short isoform of G9a dubbed as hG9a(S) and lacks the amino terminal part of isoform-a ${ }^{19,40}$.The mouse G9a (mG9a) is composed of 1229 amino acids, and its composition is closer to isoform-a of human G9a than isoform-b (Figure 1C). Following co-transfection of H1299 cells with p53 alone or in the presence of hG9a or mG9a with three different reporters (Bax-luc, PUMA-luc and p21luc) we measured luciferase activity in each case (Fig. 1 D, upper). Expression of p53 and G9a proteins was monitored by western blotting (Fig. 1D, bottom). Again, the results of luciferase assay showed the difference between human and mouse G9a towards p53-dependent activation of the reporters (Figure 1D). Specifically, hG9a(S) augmented p53 transcriptional activity at BAX, PUMA, and p21 promoters, while the expression of mG9a suppressed the activity of these reporters. Similar results were also obtained for HCT116 (p53 $3^{+/+}$and $\left.\mathrm{p} 53^{-/-}\right)$colorectal cancer cells, demonstrating that hG9a co-activates transcription in the p53-dependent manner (Supplementary 1). Furthermore, the regulation of p53 by hG9a was promoter-dependent, giving maximum increase for the PUMA reporter (1.8-fold over p53 alone) and modest 1.2fold activation for Bax and p21 (Fig. 1 D). This result indicates that hG9a acts on p53 in the gene-specific manner.

To corroborate these results, we performed Western blotting on similarly transfected H1299 cells before and after genotoxic stress induced by doxorubicin (Fig. 1E). Expression of the 
endogenous levels of Puma and p21 proteins was measured as functions of p53 and hG9a or mG9a status. While p21 protein expression levels clearly depended on the presence of doxorubicin (Fig. 1F, upper), they were not significantly affected (1.3-fold) by the presence of hG9a. However, the sample with ectopic p53 and mG9a exhibited a 3-fold decrease in expression of p21 after doxorubicin compared to p53 alone (Fig. 1F, upper). In contrast, a significant 2.2-fold increase in the protein level of Puma was observed in the sample expressing p53 and hG9a(S) after doxorubicin treatment (Fig. 1E and 1F, lower panel). Further, mG9a efficiently (2.5-fold) down-regulated the level of Puma after doxorubicin. Collectively, these results suggest that hG9a, unlike its mouse counterpart, activates p53-dependent transcription, resulting in the augmented Puma expression after doxorubicin treatment.

\section{Human G9a splice variants and its homolog, GLP, upregulate Puma expression}

In cells, G9a was shown to form a complex with its homolog, GLP (Collins R and Cheng X. Nucl. Acids Res. (2010) 38 (11): 3503-3511). To further complicate the picture, the hG9a gene product is present in two isoforms $(\mathrm{G} 9 \mathrm{a}(\mathrm{S})$ and $\mathrm{G} 9 \mathrm{a}(\mathrm{L}))$. Therefore, we wanted to determine whether the long isoform of hG9a and GLP participated in p53 regulation in a similar to hG9a(S) fashion. To address this question we employed human lung carcinoma cell line H460, which harbours wild type p53. These cells were transfected with the indicated plasmids and treated or not treated with doxorubicin to elicit p53 response. Expression levels of endogenous p21 and Puma genes was analyzed by qRT-PCR (Fig. 2A). The transfection efficiency was monitored by western blotting using specific antibodies (Fig. 2 B). Both isoforms of hG9a and GLP facilitated p53-dependent transcription of PUMA (Fig. 2B, lower panel). However, in case of $\mathrm{p} 21$, G9a proteins behaved differently, i.e. whereas hG9a(S) and GLP further stimulated p53 activation, hG9a(L) did not additionaly activate $\mathrm{p} 21$ compared to p53 alone (Fig. 2A, upper 
panel). Taken together, these results suggest that hG9a co-activated transcription with endogenous p53. Similar outcome was observed when the expression of p21 and PUMA was analyzed in H460 cells by luciferase assay (Supplementary 2).

So far, our results demonstrated the positive role of overexpressed hG9a in p53 transcriptional activity in several human cancer cell lines. To validate our observations in a more physiological setting we used shRNA against G9a (shG9a) to attenuate expression of the endogenous hG9a (Fig. 2C). Importantly, the two preparations of shRNA-G9a that were used, one was a mix of four different shRNAs and another was single shRNA, both down-regulated G9a expression. However, the pool of shRNAs was more efficient and hence was used in the subsequent experiment. H1299 cells transfected with control or p53 expression vector in the presence of absence of shRNA-hG9a were treated or non-treated with doxorubicin and subsequently were analysed for p21 and Puma expression by qRT-PCR (Fig. 2D). Attenuation of G9a expression resulted in 2-fold suppression of PUMA expression compared to cells with unaffected levels of hG9a (Figure 2D). Upon doxorubicin treatment, cells with shRNA-G9a showed 1.6-fold reduction in p53-dependent activation of PUMA. In contrast, we did not observe any significant effect of shRNA-G9a on the p21 gene induction. These data suggest that G9a specifically cooperates with p53 to regulate PUMA expression in response to genotoxic stress.

\section{hG9a affects p53 activity by the methylation-independent mechanism}

Next, we wanted to elucidate different effects of hG9a and mG9a on the molecular level. Previously, mG9a was shown to inhibit p53 via di-methylation of p53 on lysine K373, which ablated both DNA binding and transcriptional activities of $\mathrm{p} 53^{27}$. Therefore, we conducted a luciferase assay experiment where we compared the effects of transfected human and mouse G9a proteins on wild-type p53 and methylation-deficient mutant p53 (K373R). H1299 cells 
were also transfected with the PUMA luciferase reporter gene (Fig. 3 A). Unexpectedly, the results showed that $\mathrm{hG9a(S)}$ augmented the activity not only of wild-type p53 but also its unmethylatable mutant (K373R). In contrast, mG9a showed a strong negative effect on transcription only in the presence of wild-type p53. The mG9a-imposed repression was significantly alleviated when the mutant p53 (K373R) was used (Fig. 3A, upper). This effect was not due to the attenuated level of p53 expression, because western blotting showed comparable amounts of p53 in each case (Fig. 3A, bottom). Because the activity of G9a can be manipulated pharmacologically with a small molecule, BIX-01294 (Kubicek S. et al, Mol Cell. 2007 Feb 9;25(3):473-81), we wanted to assess its importance for regulation of p53-dependent transcription (Fig. 3B). To this end, HCT116 p53-positive cells transfected with PUMAluciferase reporter and either hG9a or mG9a were treated or not treated with G9a-specific inhibitor, BIX-01294, before measuring luciferase activity. The results shown in Figure 3B clearly demonstrate that inhibition of hG9a KMT activity did not have any effect on its ability to activate p53-dependent transcription. On the contrary, treatment with BIX-01294 of mG9aexpressing cells dramatically alleviated repression (6-fold) of p53. Importantly, BIX-01294 did not affect transcription of the PUMA-luciferase reporter in the absence of p53 (Fig. 3B). Collectively, these results strongly suggest that hG9a facilitates the activity of p53 in the methylation-independent manner, whereas mG9a blunts the activity of p53 via methylation on K373 as previously reported ${ }^{27}$.

\section{hG9a cooperates with CBP to promote p53 activity}

To make the first step in elucidation of the molecular mechanism by which hG9a enhances the activity of p53 we decided to assess the effect of hG9a on p53 binding to the target gene promoters by ChIP assay (Fig. 4A, B). To address this question H460 cells were transfected with either $\mathrm{hG} 9 \mathrm{a}(\mathrm{S})$ or $\mathrm{hG} 9 \mathrm{a}(\mathrm{L})$ and treated or not treated with doxorubicin to elicit $\mathrm{p} 53$ response. ChIP assay was performed using the anti-p53 antibody. As shown in Figure 4A, even 
in the absence of DNA damage overexpression of both hG9a isoforms augmented p53 binding to the promoter of PUMA (4.5-fold and 2-fold, respectively) compared to the DNA binding level of p53 alone (Fig. 4 B, lower). However, this effect was very modest (1.5-fold) in case of the p21 promoter binding (Fig. 4B, upper). Upon doxorubicin treatment, ectopic hG9a(S) and, to a lesser extent, hG9a(L) further increased p53 binding to both p21 and PUMA promoters. Similar effect of hG9a(S) on p53 binding was also observed in H1299 cells transfected with p53 in the case of PUMA promoter, but not in the case of p21 (Supplementary 3). Importantly, mG9a repressed p53 binding to PUMA, but had no effect on p53 binding to the promoter of p21 (Supplementary 3). Thus, our ChIP results suggest that hG9a enhances p53 binding to the PUMA promoter in both H1299 and H460 cells.

It is well established that p53 regulates transcription of its target genes predominantly via recruitment of specific coactivators (Sullivan KD et al., Biochim Biophys Acta. 2012 Apr;1825(2):229-44). One of the focal coactivators for p53 is histone acetyltransferase (HAT) p300/CBP (Brooks CL, Gu W. Protein Cell. 2011 Jun;2(6):456-62). Moreover, studies from Stallcup's group (Lee DY et al, J Biol Chem. 2006 Mar 31;281(13):8476-85; Bittencourt D. et al., Proc Natl Acad Sci U S A. 2012 Nov 27;109(48):19673-8) suggest that G9a controls transcription of a subset of Glucocorticoid Receptor-dependent genes by recruiting p300. Having considered these facts, we hypothesized that p53 physically interacts with hG9a and the latter facilitates additional recruitment of CBP to the PUMA promoter. To test this hypothesis we firstly determined whether endogenous p53 and hG9a physically interact in HEK293F cells treated with doxorubicin. Cell lysates were immunoprecipitated using either anti-p53 or anti-IgG (negative control) antibodies. The immunoprecipitates were examined by Western blotting with anti-p53 and anti-G9a antibodies. The endogenous hG9a protein (S and L) was specifically co-immunoprecipitated with $\mathrm{p} 53$, but not with the control IgG antibody 
(Fig. 4C). This result confirms the existence of physical interaction between endogenous hG9a and $\mathrm{p} 53$.

Because G9a was reported to localize both to the nucleus and cytoplasm we wanted to determine in which cellular compartment the p53-hG9a interaction takes place. To address this question H1299 cells were co-transfected with Flag-hG9a and p53-GFP plasmids and were analysed by indirect immunofluorescence microscopy (Fig. 4D). As can be judged from Figure 4D, both p53 and hG9a were predominantly localised to the nucleus, although a small fraction of hG9a was also present in the cytoplasm. Based on these results we concluded that the p53hG9a interaction likely takes place in the nucleus and thus may have functional effect on transcription.

Next, we questioned whether hG9a is associated with CBP. To avoid possible interference from p53, which is known to also bind CBP () we used H1299 (p53-/-) cells. Upon transfection with CBP and Flag-hG9a plasmids, cell were treated with doxorubicin and subsequently subjected to immunoprecipitation with Flag-specific antibody (Fig. 4E). Anti-IgG serum was used as negative control. The resulting immunoprecipitates were then analysed by anti-FLAG and antiCBP antibodies. We found that CBP was coimmunoprecipitated with FLAG-hG9a using Flag antibody but not with non-specific IgG (Figure 5C right panel). Hence, we concluded that hG9a interacts with CBP in the absence of $\mathrm{p} 53$.

Functional recruitment of $\mathrm{CBP}$ to gene promoters is manifested in increased acetylation of histone $\mathrm{H} 4$ (H4ac) (refs). Therefore, we examined the acH4 status of the PUMA promoter as a function of hG9a (Fig. 4F). Thus, ChIP assay was performed in H460 (p53 +/+) cells transfected with either empty vector or hG9a(S) expression plasmid. To induce DNA damage cells were treated with doxorubicin. Results of the experiment revealed that upon DNA damage the presence of hG9a(S) correlated with increased acetylation of histone $\mathrm{H} 4$ in the promoter 
region of PUMA (Fig. 4F), suggesting that the hG9a-CBP interaction is functional.

Lastly, we investigated whether this interaction affects p53-dependent transcription (Fig. 4G). Specifically, HCT116 cells $\left(\mathrm{p} 53^{+}\right.$and $\left.\mathrm{p} 53^{-}\right)$, were transfected individually with plasmids expressing hG9a, CBP, or their combination and with the PUMA-luciferase reporter followed by DNA damage induced by doxorubicin (Fig. 4G). Luciferase assay results showed that both hG9a and CBP co-activated transcription of the reporter with p53 after doxorubicin. Importantly, when hG9a and CBP were co-expressed together with p53 we observed further increase in luciferase activity when compared to the individual levels of either p53 and hG9a or p53 and CBP activation (1.7-fold). This result indicates that hG9a and CBP cooperate to augment p53-dependent transcription.

\section{Expression of hG9 augments p53-dependent cell death}

To evaluate the physiological significance of hG9a-dependent effect on p53-dependent activation of PUMA we performed the clonogenic assay using H460 p53-positive cells ectopically expressing either hG9a or mG9a with and without doxorubicin treatment (Fig. 5A). DNA damage itself decrease the number of colonies in control cells 2-fold (Fig. 5A, lower panel). Importantly, expression of hG9a in H460 cells even withhout DNA damage resulted in a reduction of the number of colonies, which was furhter substantiated by genotoxic stress. In striking contrast to hG9a, forced expression of mG9a even slightly increased the number of colonies after doxorubicin, arguing that mG9a inhibited the activity of p53. Furthermore, to examine the cell cycle distribution of these cells treated or not treated with doxorubicin we conducted fluorescence-activated cell sorting (FACS) analysis (Fig. 5B). As shown in Figure 5B, we did not observe any dramatic difference between the pCDNA3 control and hG9aexpressing cells after doxorubicin. The only significant difference specific for hG9a-expressing cells was the level of cell death (Fig.5C), which was the highest for that time of cells (compare 
$24 \%$ vs $17.8 \%$ and $16 \%$ for pCDNA3 and mG9a cells, respectively). In summary, we established that forced expression of hG9a correlates with increased cell death.

\section{Correlation between p53 and hG9a expression is associated with better survival}

To provide the biological significance to our observations on positive effect of hG9a on p53 we analysed clinical gene expression arrays data for correlations between the co-expression of hG9a and p53 and the survival rates of cancer patients (Antonov AV, Krestyaninova et al, Oncogene, 2013) (Fig. 6). Initially, we wanted to assess the importance of hG9a as a prognostic marker. Importantly, we found that high expression of hG9a positively correlated with better survival of stage I-II lung cancer patients (Fig. 6A). Next, using GINT software (available at bioprofiling.de, Antonov AV. Nucleic Acids Res. 2011 Jul;39(Web Server issue):W323-7.), which divides the expression data for the two genes of interest into two cohorts, one with no or strong negative correlation and the other one with strong correlation in their expression and links to Kaplan-Meier survival probability estimates (Fig. 6B). Thus, using different datasets we found that correlation in the expression between hG9a and p53 was associated with better survival of lung cancer patients $(\mathrm{p}=0.002)$ (Fig. 6B and Supplementary 4). Furthermore, we also found that high expression of G9a (EHMT2) and p53 correlated with better survival of patients with colon $(\mathrm{p}=0.027)$ and ovarian cancer $(\mathrm{p}=0.0005)$ (Supplementary 5). These results highlight the biological significance of the hG9a-p53 association in several types of cancer.

\section{DISCUSSION}

p53 is one of the major tumour suppressors in vertebrates. The TP53 gene is conserved among different species, however the spectra of its post-translational modifications are quite different depending on the organism (Marouco et al, 2013; Pant V and Lozano G., Genes \& Dev. 2014. 28: 1739-1751). For example, lysine methylation on K372 discovered for the human p53 protein, is absent in the mouse one (Campaner S. et al., Mol Cell. 2011 Aug 19;43(4):681-8; 
Lehnertz B, Mol Cell. 2011 Aug 19;43(4):673-80). Further, sumoylation on K386 in the human p53 does not occur in its mouse paralogue (Marouco et al 2013 and references therein). In line with this is our observation that hG9a, unlike its mouse counterpart, cannot efficiently methylate p53 on K373 (Huang et al, JBC and data not shown).

Our extensive studies, including luciferase assays, mRNA evaluation, and Western blotting in several cell lines establishes human G9a as a p53 co-activator for at least a subset of genes, including the pro-apoptotic PUMA. Several studies support the hypothesis that hG9a, depending on the chromatin context and protein-protein interactions, can either repress or activate transcription thus serving as a bivalent regulator of transcription. Importantly, transcriptional activation by G9a is exerted in a methyltransferase-independent manner (C.P. Chaturvedi et al., Proc. Natl. Acad. Sci. USA, 106 (2009), pp. 18303-18308; Bittencourt D. et al., Proc Natl Acad Sci U S A. 2012 Nov 27;109(48):19673-8.). Accordingly, pharmacological inactivation of G9a with specific small molecules did not affect transcriptional activation of genes studied, which is in agreement with the results of our study (Fig. 3B). Then, what is the mechanism of G9a-mediated activation? A possible explanation to this phenomenon relies on the fact that G9a is associated with multiple protein complexes (e.g. Snail-G9a-DNMT1, REST-CDYL-G9a, G9a-p300-CARM1-GRIP1-MED), which play both positive and negative roles in transcription (Dong C. et al., Cancer Cell. 2013 Mar 18;23(3):316-31; Mulligan P. et al., Mol Cell. 2008 Dec 5;32(5):718-26; Shankar SR et al., Epigenetics. 2013 Jan;8(1):16-22.). In our study, we detected CBP as a binding partner of hG9a (Fig. 4), which is recruited to the promoter of PUMA to acetylatye histone H4 (Fig. 4F). Similar results were obtained for GRdependent transcription where G9a recruited p300 to a subset of responsive genes (Lee DY., J Biol Chem. 2006 Mar 31;281(13):8476-85). Another study has shown that hG9a upregulated transcription of the p21 gene upon DNA damage via interaction with another HAT, PCAF (Oh ST et al., FEBS Lett. 2014 Mar 3;588(5):685-91). Collectively, these reports suggest that G9a 
mediates its activation functions via recruitment of HATs in the methylation-independent fashion.

It should be noted however, that the positive effect of hG9a on p53 is gene-specific as we did not detect much effect of hG9a on p53-dependent activation of p21 (Fig. 1D and 1E). Currently, we do not have an explanation to such selectivity of hG9a action, yet other studies support our observation. For example, treatment of Smyd2-deficient cells with an inhibitor of G9a, BIX01294, selectively activated the downstream genes of p53 signalling pathway, including p21 and DOR, but not PUMA (Fan JD et al., PLoS One. 2015 Jan 6;10(1):e0116782).

Another begging question that remains to be answered is why the human and mouse orthologues of G9a have diametrically opposed effects on p53? We speculate that such a drastic difference is mediated by structural differences in these proteins. The human G9a (EHMT2) gene is present in cells as $\mathrm{hG} 9 \mathrm{a}(\mathrm{L})$ and $\mathrm{hG} 9 \mathrm{a}(\mathrm{S})$ isoforms. The longest one resembles more the structure of the mG9a protein, which is the product of NG36-G9a transcript (Fig. 1C and Shankar SR, et al. 2013). Previous studies have revealed differences in the sub-cellular distribution of the NG36-G9a and G9a proteins. The NG36-G9a is mostly located in the nucleus, whereas G9a localizes both to the cytoplasm and nucleus ${ }^{40}$. In fact, we also detected small amounts of hG9a in the cytoplasm (Fig. 4D). Additionally, the amino-terminus of mG9a contains eight Glycine-Arginine (G-R) repeats. The G-R repeats function as NLS and are found in proteins involved in gene expression, RNA splicing and translation (Thandapani P., Mol Cell. 2013 Jun 6;50(5):613-23 and references therein). However, in our case, mG9a blunted transcription by attenuating p53 binding to the promoters of its target genes via methylation on K373 (Supplementary 3). The question of why the human and mouse G9a proteins behave so differently towards p53 requires additional investigation, which is beyond the scope of this study. 
Recently, Huang et $\mathrm{al}^{27}$ has reported that allegedly human G9a protein also inhibited p53 activity by methylating it on K373, which contradicts the results of our study. The most obvious explanation to this discrepancy is that Huang et al may have used the mouse version of G9a, which is similar in size with the $\mathrm{hG} 9 \mathrm{a}(\mathrm{L})$ form (Fig. 1D). Another reason behind this disagreement may be a result of using different cell lines. For example, Huang et al. (2010) used MCF7 (Human Breast Adenocarcinoma) cells ${ }^{27}$, where our bioinformatics analysis failed to reveal the correlation between G9a expression and survival of cancer patients (data not shown).

It should be emphasized that the positive correlation between the p53-G9a expression and better survival of patients is tumor-specific. Our bioinformatics results, in addition to breast cancer, also failed to reveal any positive correlations for lymphoma, melanoma and prostate cancers (data not shown). Despite the wealth of data on the oncogenic role of G9a we found that at least in the initial stages of lung adenocarcinoma elevated expression of hG9a correlated with better survival rates (Fig. 6A), which fits the description of G9a as a bivalent regulator of transcription. As an indirect evidence of this functional link is the fact that in tumour cell lines which harbour wild-type p53 the level of hG9a expression is usually quite low and tumour cells exhibit increased apoptosis when the levels of both p53 and hG9a are elevated (Fig. 5 and data not shown). Furthermore, increased levels of p53 and G9a also correlated with better prognosis for colon and ovarian cancer patients (Supplementary 4).

In summary, we reported here a novel role for human G9a as a gene-specific p53 transcriptional coactivator. In drastic contrast to its mouse counterpart, hG9a activated p53dependent transcription in the methylation-independent manner through the recruitment of CBP and subsequent acetylation of histones in the promoters of p53-dependent genes (Fig. 7). These findings raise an important question of the efficacy of G9a-specific pharmacological inhibitors as therapeutic tools to treat p53-positive tumors. 


\section{ACKNOWLEDGMENTS}

We need to acknowledge people, who gave us plasmids (M Stallcup) and other reagents!

\section{REFERENCES}

1. Marouco D, Garabadgiu A, Melino G, Barlev N. Lysine-specific modifications of p53: a matter of life and death? Oncotarget 2013;4(10):1556-1571. Available at:

http://www.ncbi.nlm.nih.gov/pmc/articles/PMC3858545/. Accessed October 21, 2014.

2. Vousden KH, Ryan KM. P53 and Metabolism. Nat. Rev. Cancer 2009;9(10):691-700. doi:10.1038/nrc2715.

3. Kruse J-P, Gu W. Modes of p53 regulation. Cell 2009;137(4):609-22. doi:10.1016/j.cell.2009.04.050.

4. Greenberg RA, Chin L, Femino A, et al. Short Dysfunctional Telomeres Impair Tumorigenesis in the INK4a 回 2 / 3 Cancer-Prone Mouse. 1999;97:515-525.

5. Stiewe T. The p53 family in differentiation and tumorigenesis. 2007;7(March):165168.

6. Michalak EM, Vandenberg CJ, Delbridge ARD, et al. Apoptosis-promoted tumorigenesis: gamma-irradiation-induced thymic lymphomagenesis requires Pumadriven leukocyte death. Genes Dev. 2010;24(15):1608-13. doi:10.1101/gad.1940110.

7. Basu A, Haldar S. The relationship between Bcl2, Bax and p53 : consequences for cell cycle progression and cell death. 1998;4(12):1099-1109.

8. Oda E. Noxa, a BH3-Only Member of the Bcl-2 Family and Candidate Mediator of p53-Induced Apoptosis. Science (80-. ). 2000;288(5468):1053-1058.

doi:10.1126/science.288.5468.1053.

9. Abdelalim EM, Tooyama I. Knockdown of p53 suppresses Nanog expression in embryonic stem cells. Biochem. Biophys. Res. Commun. 2014;443(2):652-7. doi:10.1016/j.bbrc.2013.12.030.

10. Spike BT, Wahl GM. p53, Stem Cells, and Reprogramming: Tumor Suppression beyond Guarding the Genome. Genes Cancer 2011;2(4):404-19. doi:10.1177/1947601911410224.

11. Bai L, Zhu W. p53 : Structure, Function and Therapeutic Applications. 2006:141-153. 
12. Laine a, Ronai Z. Regulation of p53 localization and transcription by the HECT domain E3 ligase WWP1. Oncogene 2007;26(10):1477-83.

doi:10.1038/sj.onc.1209924.

13. Muller P a J, Vousden KH. P53 Mutations in Cancer. Nat. Cell Biol. 2013;15(1):2-8. doi:10.1038/ncb2641.

14. Fernandez-Fernandez MR, Sot B. The relevance of protein-protein interactions for $\mathrm{p} 53$ function: the CPE contribution. Protein Eng. Des. Sel. 2011;24(1-2):41-51. doi:10.1093/protein/gzq074.

15. Dai C, Gu W. P53 Post-Translational Modification: Deregulated in Tumorigenesis. Trends Mol. Med. 2010;16(11):528-36. doi:10.1016/j.molmed.2010.09.002.

16. Smeenk L, van Heeringen SJ, Koeppel M, et al. Role of p53 serine 46 in p53 target gene regulation. PLoS One 2011;6(3):e17574. doi:10.1371/journal.pone.0017574.

17. Scoumanne and Chen. Protein methylation: a new regulator of the p53 tumor suppressor. Histol Histopathol 2008;23(9):1143-1149. Available at: http://www.ncbi.nlm.nih.gov/pmc/articles/PMC2762123/.

18. Chang Y, Zhang X, Horton JR, et al. Structural basis for G9a-like protein lysine methyltransferase inhibition by BIX-01294. Nat. Struct. Mol. Biol. 2009;16(3):312-7. doi:10.1038/nsmb.1560.

19. Tachibana M, Ueda J, Fukuda M, et al. Histone methyltransferases G9a and GLP form heteromeric complexes and are both crucial for methylation of euchromatin at H3-K9. Genes Dev. 2005;19(7):815-826. doi:10.1101/gad.1284005.

20. Tachibana M, Sugimoto K, Fukushima T, Shinkai Y. Set domain-containing protein, G9a, is a novel lysine-preferring mammalian histone methyltransferase with hyperactivity and specific selectivity to lysines 9 and 27 of histone H3. J. Biol. Chem. 2001;276(27):25309-17. doi:10.1074/jbc.M101914200.

21. Shinkai Y, Tachibana M. H3K9 methyltransferase G9a and the related molecule GLP. Genes Dev. 2011;25(8):781-8. doi:10.1101/gad.2027411.

22. Kubicek S, O’Sullivan RJ, August EM, et al. Reversal of H3K9me2 by a SmallMolecule Inhibitor for the G9a Histone Methyltransferase. Mol. Cell 2007;25(3):473481. doi:10.1016/j.molcel.2007.01.017.

23. Weiss $\mathrm{T}$, Hergeth $\mathrm{S}$, Zeissler $\mathrm{U}$, et al. Histone $\mathrm{H} 1$ variant-specific lysine methylation by G9a/KMT1C and Glp1/KMT1D. Epigenetics Chromatin 2010;3(1):7. doi:10.1186/1756-8935-3-7.

24. Trojer P, Zhang J, Yonezawa M, et al. Dynamic Histone H1 Isotype 4 Methylation and Demethylation by Histone Lysine Methyltransferase G9a/KMT1C and the Jumonji Domain-containing JMJD2/KDM4 Proteins. J Biol Chem. 2009 Mar 27; 284(13) 8395-8405. 2009;27(13):8395-8405. 
25. Shankar SR, Bahirvani AG, Rao VK, Bharathy N, Ow JR, Taneja R. G9a, a multipotent regulator of gene expression. Epigenetics 2013;8(1):16-22. doi:10.4161/epi.23331.

26. Rathert P, Dhayalan A, Murakami M, et al. NIH Public Access. 2009;4(6):344-346. doi:10.1038/nchembio.88.Protein.

27. Huang J, Dorsey J, Chuikov S, et al. G9a and Glp methylate lysine 373 in the tumor suppressor p53. J. Biol. Chem. 2010;285(13):9636-41. doi:10.1074/jbc.M109.062588.

28. Ueda J, Tachibana M, Ikura T, Shinkai Y. Zinc finger protein Wiz links G9a/GLP histone methyltransferases to the co-repressor molecule CtBP. J. Biol. Chem. 2006;281(29):20120-8. doi:10.1074/jbc.M603087200.

29. Tachibana M, Sugimoto K, Nozaki M, et al. G9a histone methyltransferase plays a dominant role in euchromatic histone $\mathrm{H} 3$ lysine 9 methylation and is essential for early embryogenesis. Genes Dev. 2002;16(14):1779-1791. doi:10.1101/gad.989402.

30. Purcell DJ, Jeong KW, Bittencourt D, Gerke DS, Stallcup MR. A distinct mechanism for coactivator versus corepressor function by histone methyltransferase G9a in transcriptional regulation. J. Biol. Chem. 2011;286(49):41963-41971. doi:10.1074/jbc.M111.298463.

31. Chaturvedi C-P, Somasundaram B, Singh K, et al. Maintenance of gene silencing by the coordinate action of the H3K9 methyltransferase G9a/KMT1C and the H3K4 demethylase Jarid1a/KDM5A. Proc. Natl. Acad. Sci. 2012;109(46):1-6. doi:10.1073/pnas.1213951109.

32. Lee DY, Northrop JP, Kuo M-H, Stallcup MR. Histone H3 lysine 9 methyltransferase G9a is a transcriptional coactivator for nuclear receptors. J. Biol. Chem. 2006;281(13):8476-8485. doi:10.1074/jbc.M511093200.

33. Oh ST, Kim KB, Chae YC, Kang JY, Hahn Y, Seo SB. H3K9 histone methyltransferase G9a-mediated transcriptional activation of p21. FEBS Lett. 2014;588(5):685-691. doi:10.1016/j.febslet.2014.01.039.

34. Bittencourt D, Wu D-Y, Jeong KW, et al. G9a functions as a molecular scaffold for assembly of transcriptional coactivators on a subset of glucocorticoid receptor target genes. Proc. Natl. Acad. Sci. U. S. A. 2012;109(48):19673-8. doi:10.1073/pnas.1211803109.

35. Duan Z, Zarebski A, Montoya-durango D, Grimes L, Horwitz M, Grimes HL. Gfi1 Coordinates Epigenetic Repression of p21 Cip / WAF1 by Recruitment of Histone Lysine Methyltransferase G9a and Histone Deacetylase 1. 2005;25(23):10338-10351. doi:10.1128/MCB.25.23.10338.

36. Nishio H, Walsh MJ. CCAAT displacement protein/cut homolog recruits G9a histone lysine methyltransferase to repress transcription. Proc. Natl. Acad. Sci. U. S. A. 2004;101(31):11257-11262. doi:10.1073/pnas.0401343101. 
37. Kim JK, Estève PO, Jacobsen SE, Pradhan S. UHRF1 binds G9a and participates in p21 transcriptional regulation in mammalian cells. Nucleic Acids Res. 2009;37(2):493505. doi:10.1093/nar/gkn961.

38. Kim K-B, Son H-J, Choi S, et al. H3K9 methyltransferase G9a negatively regulates UHRF1 transcription during leukemia cell differentiation. Nucleic Acids Res. 2015:115. doi:10.1093/nar/gkv183.

39. Dai C, Shi D, Gu W. Negative regulation of the acetyltransferase TIP60-p53 interplay by UHRF1 (ubiquitin-like with PHD and RING finger domains 1). J. Biol. Chem. 2013;288(27):19581-19592. doi:10.1074/jbc.M113.476606.

40. Brown S, Campbell R, Sanderson C. Novel NG36/G9a gene products encoded within the human and mouse MHC class III regions. Mamm Genome. 2001;12(12):916-24. Available at: http://www.ncbi.nlm.nih.gov/pubmed/11707778.

41. Chen L, Li Z, Zwolinska AK, et al. MDM2 recruitment of lysine methyltransferases regulates p53 transcriptional output. EMBO J. 2010;29(15):2538-2552. doi:10.1038/emboj.2010.140.

42. Mauger O, Klinck R, Chabot B, Muchardt C, Allemand E, Batsche E. Alternative splicing regulates the expression of G9A and SUV39H2 methyltransferases, and dramatically changes SUV39H2 functions. Nucleic Acids Res. 2015;43(3):1869-1882. doi:10.1093/nar/gkv013.

43. Estève P-O, Patnaik D, Chin HG, Benner J, Teitell M a, Pradhan S. Functional analysis of the N- and C-terminus of mammalian G9a histone H3 methyltransferase. Nucleic Acids Res. 2005;33(10):3211-23. doi:10.1093/nar/gki635.

44. Bouleau S, Pârvu-Ferecatu I, Rodriguez-Enfedaque A, et al. Fibroblast Growth Factor 1 inhibits p53-dependent apoptosis in PC12 cells. Apoptosis 2007;12(8):1377-1387. doi:10.1007/s10495-007-0072-x.

45. Liu Y, Kulesz-Martin M. p53 protein at the hub of cellular DNA damage response pathways through sequence-specific and non-sequence-specific DNA binding. Carcinogenesis 2001;22(6):851-860. doi:10.1093/carcin/22.6.851.

46. Robin P, Fritsch L, Philipot O, Svinarchuk F, Ait-Si-Ali S. Post-translational modifications of histones $\mathrm{H} 3$ and $\mathrm{H} 4$ associated with the histone methyltransferases Suv39h1 and G9a. Genome Biol. 2007;8(12):R270. doi:10.1186/gb-2007-8-12-r270.

\section{METHODS}

Plasmid constructs. pBV-Luc-PUMA, pCDNA3-p53, pGL3-p21 (Luc), pCDNA3-FLAGhG9a(S), pCDNA3-FLAG- hG9a(L), pCDNA3-FLAG-hGLP, pCDNA3-SMYD2, pCDNA3- 


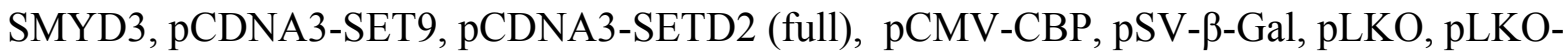
shG9a \#5, pLKO-shG9a \#6, pLKO-shG9a \#8, pLKO-shG9a \#9, and, pLKO-shG9a \#10.

Cell culture and transient transfection. Cells were cultured in prepared DMEM and incubated at $37^{\circ} \mathrm{C}$ with $5^{\circ} \mathrm{CO}_{2}$. Then, after $24-48$ hours the media was renewed based on the cells' confluency. The cells were cultured and grown till the confluency reach a target confluency $(70-80 \%)$, and the transfection was carried out according protocol provided with Turbofect transfection reagent protocol (Thermo Scientific, \# R0531). Following the protocol, the dilution of plasmid was carried out with serum-free medium (Opti-MEM) (GIBCO, Life Technologies) with incubation at room temperature for 5 minutes, and required amount of Turbofect being added. The mixture (plasmid/Turbofect) has mixed well with pipetting and incubated for 15-20 minutes at room temperature to produce micelles containing DNA, and then the Turbofect/DNA mixture being added in drop-wise, and the cells are incubated $\left(37^{\circ} \mathrm{C}\right.$ with $5^{\circ} \mathrm{CO}_{2}$ ) for 24-48 hours for further growing, and then collected.

Luciferase Reporter Assay. 100,000 cells per well were seeded in 0.5ml growth media in 24well plates the day before transfection. Transfection was performed with Turbofect (Life Technologies \#R0531) using manufacturer's protocol. $1.0 \mu \mathrm{g}$ total plasmid DNA (0.3 $\mu \mathrm{g}$ luciferase construct, $0.2 \mu \mathrm{g} \beta$-galactosidase construct, and $0.5 \mu \mathrm{g}$ empty control vector or BTK construct) was used. 24 hours after transfection, media was replaced by fresh growth media and part of samples was treated with 1.0 $\mu \mathrm{M}$ Doxorubicin. 20 hours later, lysates were collected in lysis buffer and stored at $-80^{\circ} \mathrm{C}$ overnight. $(10 \mathrm{ml}$ of $\beta$-galactosidase stock solution, $20 \mathrm{mg}$ onitrophenyl- $\beta$-d-galactopyranoside (ONPG) and $35 \mu \mathrm{l} \beta$-mercaptoethanol and $100 \mu \mathrm{l} \beta$ galactosidase substrate were added to $80 \mu 1$ cell lysate (per well) in a 96-well transparent plate. This was incubated at $37^{\circ} \mathrm{C}$ for 15 minutes. $20 \mu$ l of the cell lysate were transferred to a $96-$ well opaque white plate and read in a luminometer, using luciferase kit (BioVision\#K801-200). 
Immunoblot Analysis. For lysate extraction, medium was removed and plates were washed once with 1x PBS and trypsinzed, collected and kept on ice. The cells were sonicated using Diagenode Bioruptor 2000and the cell pellet was suspended and incubated for 20 minutes on ice. Cells were ruptured by passing through a syringe 5 times or with sonication, and centrifuged for 15 minutes at $12,000 \mathrm{rpm}$ and the supernatant was transferred into tubes where $35 \mu l$ of $4 \mathrm{X}$ loading buffer was added. Protein concentrations were determined using Bradford protein assay (Fermentas). $10-20 \mu \mathrm{g}$ of total protein per sample were subjected to $10 \%$ or $6 \%$ SDS PAGE and transferred to Immobilon-P membranes (Millipore). Primary antibodies used were: $\beta$-actin (Abcam, \#ab8227), G9a (Thermo Scientific, \#MA5-14880), p53 (Calbiochem, \#Op43), FLAG (Sigma-Aldrich, \#F3165), p21 (Santa Cruz Biotechnology, \#sc-53870), PUMA (Cell Signalling, \#4976), CBP (Santa Cruz Biotechnology, \#sc-369).

Protein-protein interaction. For the protein-protein binding assays, cells were lysed in lysis buffer (PBS, $50 \mathrm{mM} \mathrm{NaCl}, \quad 1 \%$ NP-40, $1 \mathrm{mM}$ PMSF) at $4{ }^{\circ} \mathrm{C}$. Proteins were immunoprecipitated with anti-IgG (Santa Cruz Biotechnology, \# sc-2025), anti-p53, or antiFLAG antibodies overnight at $4{ }^{\circ} \mathrm{C}$, and then protein A/G agarose beads (Millipore, \#16-157) were added for $4 \mathrm{~h}$ with rotation at $4{ }^{\circ} \mathrm{C}$. Bound proteins were analyzed via Western blotting with anti-p53, anti-G9a, anti-FLAG, anti-Flag, and anti-CBP antibodies.

In vitro methylation assay. Methylation reaction was prepared by mixing $\mathrm{x} \mu 1$ of methylation buffer $\left(20 \mu 1\right.$ Tris- $\mathrm{HCl} \mathrm{pH} 8.0,50 \mathrm{mM} \mathrm{NaCl}, 0.5 \mathrm{mM}$ DTT, 10\% Glycerol, 1mM PMSF), ${ }^{3} \mathrm{H}-$ SAM (Adenosyl-L-Methionine, S-[methyl- $\left.{ }^{3} \mathrm{H}\right]$ ) (Perkin Elmer, \#NET155250UC) $1.5 \mu$ 1, p53 $0.5 \mu \mathrm{g}$, methyltransferase enzyme $0.5 \mu \mathrm{g}$. The reaction was incubated by placing it at $30^{\circ} \mathrm{C}$ for 60 minutes. The samples were loaded onto an SDS-PAGE gel and electrophoresis was applied followed by transferring on nitrocellulose membrane. The membrane was stained with $50 \mathrm{ml}$ of DB-71 (0.008\%) (4 ml Stock DB-71 (0.1\%), and $46 \mathrm{ml}$ washing solution (20 $\mathrm{ml}$ Ethanol, 5ml Glacial acetic acid)). The membrane was rinsed with $\mathrm{ddH}_{2} \mathrm{O}$, pictured, dried, and sprayed 
with autoradiography enhancer EN³HANCE (Perkin Elmer, \#6NE9701). The dried membrane was incubated overnight at $-80^{\circ} \mathrm{C}$, and then developed.

G9a Silencing. To generate cells with a stable downregualtion of G9a, an shRNA against G9a was used. To increase the efficiency of shG9a, a shG9a pool was prepared by mixing equal amount of following types: \#5, \#6, \#8,\#9, and \#10. pLKO plasmid was used as a control. The shRNA were transfected into H1299 using Turbofect following manufacturer's protocols. The medium in the plates was changed after 60 hours incubation. To examine knockdown efficiency by shG9a, a cell lysate was prepared from part of cells and used for immunoblotting. RNA was isolated from the remaining cells, cDNA was prepared, and levels of GAPDH, p21, and PUMA were assessed by qRT-PCR.

Quantitative real time PCR qRT-PCR. RNA purification, cDNA preparation and qRT-PCR were performed following the protocol. Primers used: PUMA: GACCTCAACGCACAGTACGA (FWD), CACCTAATTGGGCTCCATCT (REV). p21: CACCGAGACACCACTGGAGG (FWD), GAGAAGATCAGCCGGCGTTT (REV). GAPDH: GGGAAGGTGAAGGTCGGAGT (FWD), TTGAGGTCAATGAAGGGGTCA (REV).

ChIP Assays. ChIP assays were performed following the protocol. Briefly, $3 \times 106$ cells per sample were crosslinked with $1 \%$ formaldehyde for 15 minutes. Crosslinking was neutralised with $0.125 \mathrm{M}$ glycine, and cells were scraped in phosphate buffer saline (PBS). Chromatin was sonicated using the Diagenode Bioruptor 2000 for 15 min with 30-s pulse/pause cycles in polycarbonate tubes on ice to shear chromatin to 300 - to 600-bp fragments. Unsheared debris was spun down, and then the chromatin was incubated overnight with the appropriate antisera, concurrent with the blocking of protein G-Sepharose beads using $2.5 \%$ bovine serum albumin (BSA). Immune complexes were then precipitated using 'blocked' protein $\mathrm{G}$ beads for $4 \mathrm{~h}$ at 
$4^{\circ} \mathrm{C}$, washed three times, and then eluted. Immunoprecipitated DNA was purified and quantitative PCR was performed with $1 \mu \mathrm{l}$ of DNA. The p53 (Galbichem, \#OP43), Acetylated H4 (Upstate, \#06-599), Dimethylted H3K27 (Upstate, \#07-322), and Dimethylted H3K9 (Upstate, \#07-212) antibodies were used.

Primers used were as follows. PUMA: GCGAGACTGTGGCCTTGTGT (FWD), CGTTCCAGGGTCCACAAAGT (REV). p21: GTGGCTCTGATTGGCTTTCTG (FWD),

CTGAAAACAGGCAGCCCAAG (REV). Actin: TGGCTCAGCTTTTTGGATTC (FWD), CCGAGGATTGGAGAAGCAGT (REV).

FACS analysis. Cells were seeded in triplicate into 6-well plates and left for 24 hours before transfection. Media was changed and part of the samples was treated with doxorubicin. The cells were harvested by trypsinization. The pellets fixed, and stained with Propidium iodide (PI) buffer (50 $\mu \mathrm{g} / \mathrm{ml}$ of Propidium iodide (Sigma-Aldrich, \#P4170), $10 \mu \mathrm{g} / \mathrm{ml}$ Ribonuclease A (Sigma-Aldrich, \#R6513), PBS 1X) for 1 hour at $37^{\circ} \mathrm{C}$ while protected from light. Flow cytometry analysis was performed using Becton Dickinson FACSanto II FACSDiva 6.0 software (Becton Dickinson), and 10,000 events were recorded for each sample using for acquisition and analysis.

Colony formation assay. 500 cells were split into $60 \mathrm{~mm}$ plates in triplicates and incubated at $37^{\circ} \mathrm{C}$ and $5 \% \mathrm{CO}$. The next day cells were transfected and incubated at $37^{\circ} \mathrm{C}$ and $5 \% \mathrm{CO}_{2}$. After, 34 hours, the old media was replaced with a new media supplemented with $500 \mu \mathrm{M} / \mathrm{ml}$ of Geneticin antibiotic (Santa Cruz, \#sc-29065). The media was changed every 3 days and cells were allowed to grow at $37^{\circ} \mathrm{C}$ and $5 \% \mathrm{CO}_{2}$ for 14 days. Plates were then washed with $3 \mathrm{ml}$ PBS and fixed with $2 \mathrm{ml}$ of 10\% neutral buffered formalin (Sigma-Aldrich). Plates were incubated at room temperature for $30 \mathrm{~min}$. Formalin was removed by aspiration and plates were washed twice with PBS, and then allowed to completely air dry with the lids removed. $5 \mathrm{ml}$ of 
Staining Reagent (6.4 $\mathrm{ml}$ of PO4 buffer $(67 \mathrm{mM}), 89.6 \mathrm{ml}$ of dH2O, $4 \mathrm{ml}$ Giemsa stain (Fluka)) was finally added to the plates and they were incubated at room temperature for 5 hours. The staining solution was poured off and the plates were rinsed gently with ddH2O. Plates were allowed to air dry and the number of colonies from each plate was then counted and recorded.

\section{Indirect Immunofluorescence}

Cultures of H1299 were grown on gelatin-coated 12-well plates and co-transfected with plasmids expressing FLAG-G9a and GFP-p53 using TransIT-2020 transfection reagent (Mirus). Transfected cells were fixed with 4\% paraformaldehyde for $20 \mathrm{~min}$, permeabilized for 20 min with $0.2 \%$ Triton X-100 in PBS (pH 7.4) at room temperature. To prevent non-specific protein binding, cells were incubated with 5\% BSA in PBS for $1 \mathrm{~h}$ at room temperature. Cells were then incubated with the primary antibodies [Monoclonal ANTI-FLAG M2, diluted at 1:2,000 in 3\% BSA/PBS (Sigma Aldrich)] for 2 hours followed by secondary antibodies [Alexa Fluor 546 Donkey Anti-Mouse IgG, diluted at 1:500 in PBS (Life Technologies)] for 1 hour at room temperature. Nuclei were visualized with DAPI (4,6 diamidino- 2-phenylindole; Life Technologies). Immunofluorescence images were collected using a laser scanning confocal microscope (Leica TCS-SP5). 
A

\begin{tabular}{|c|c|c|c|c|c|}
\multicolumn{1}{r}{ N-terminus } & \multicolumn{1}{c}{ Central core } & \multicolumn{3}{c|}{ C-terminus } \\
\hline TAD & DBD & NLS & TD & NES & RD \\
$1-90$ & $102-292$ & & $323-356$ & & $363-393$ \\
\hline
\end{tabular}

(p53 monomer structure)

C

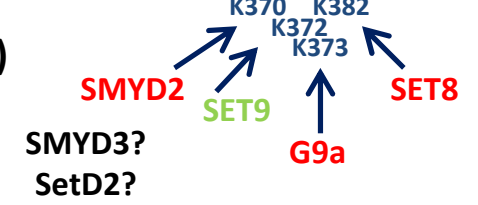

SetD2?

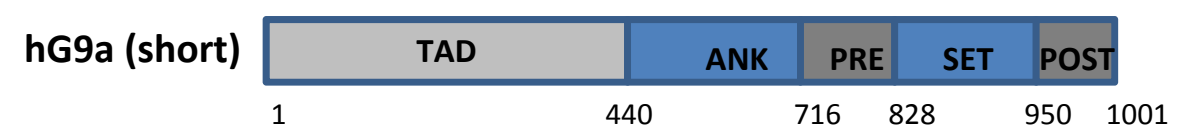

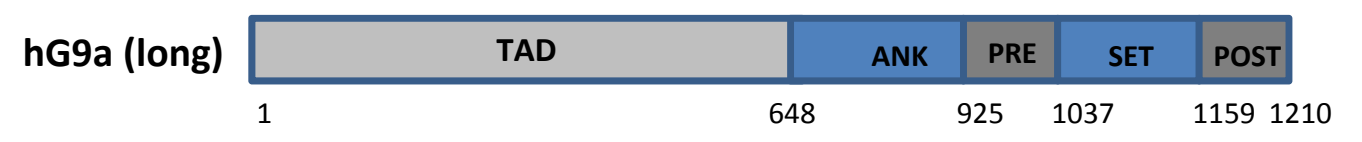

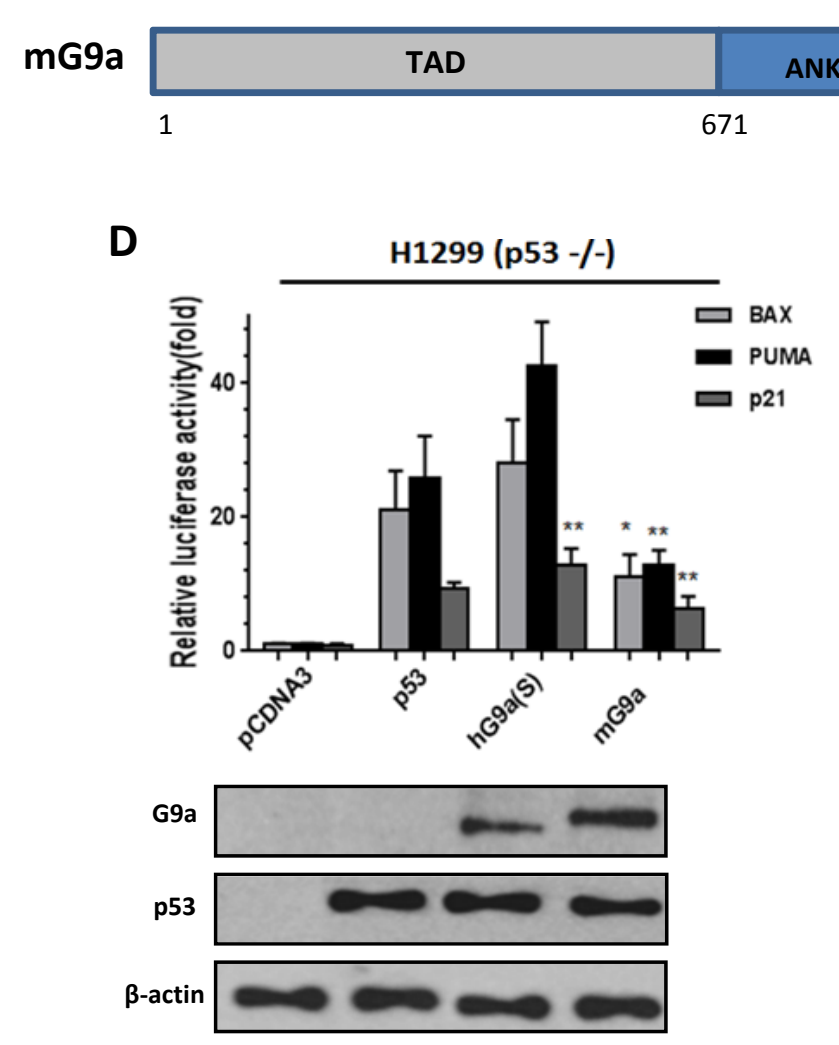

B

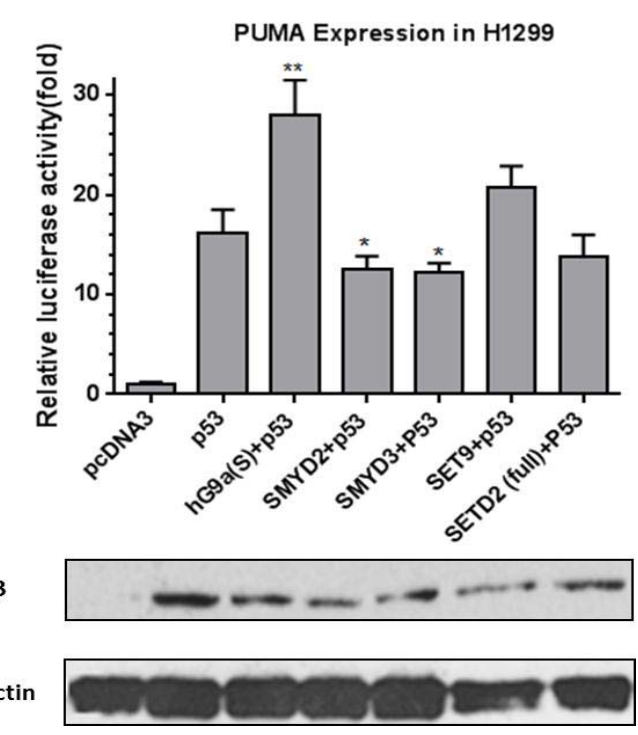

H1299 cells

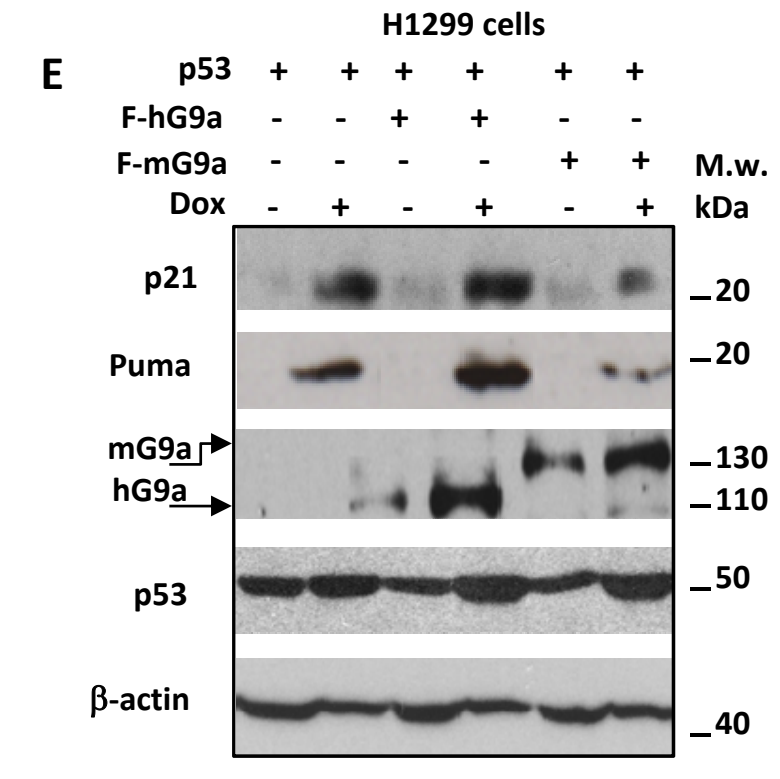

F Western blot quantification
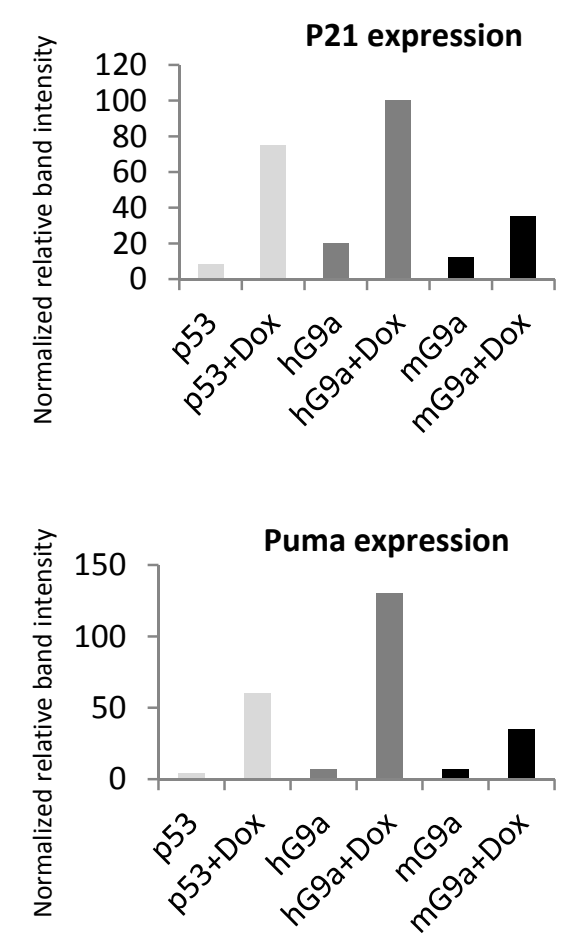

Figure 1 

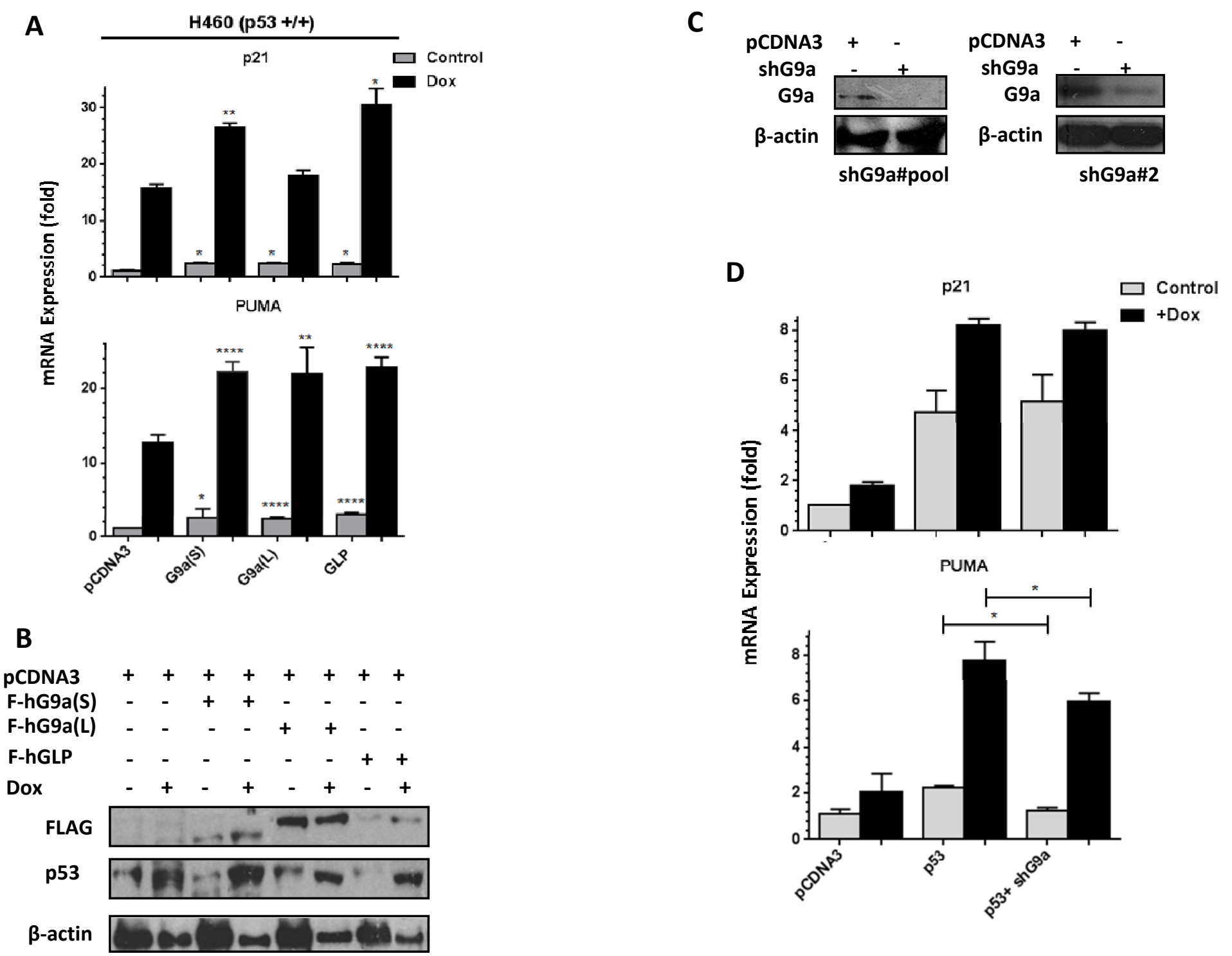
A

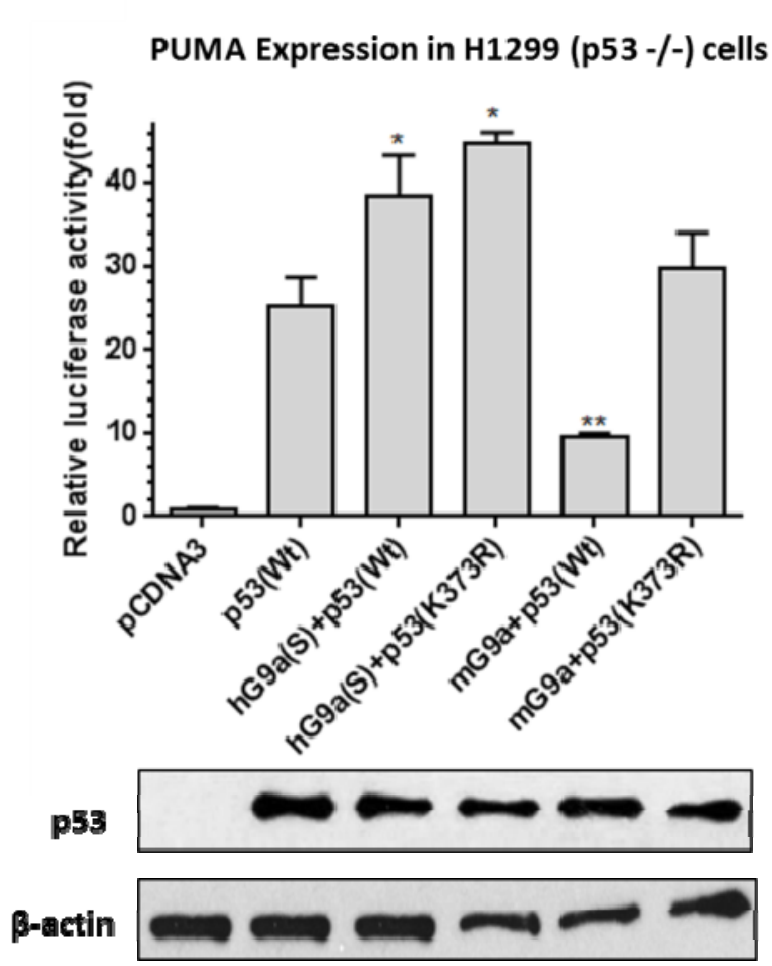

B

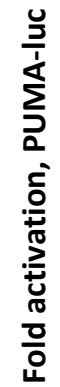

HCT116 (p53+/+) cells treated with BIX-01294

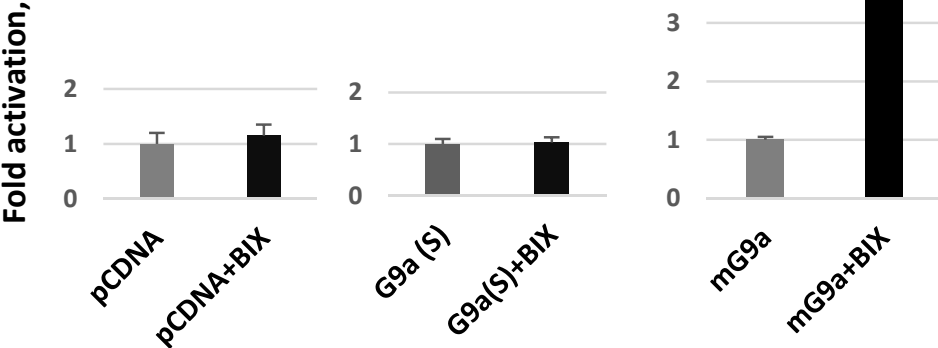

Figure 3 
A

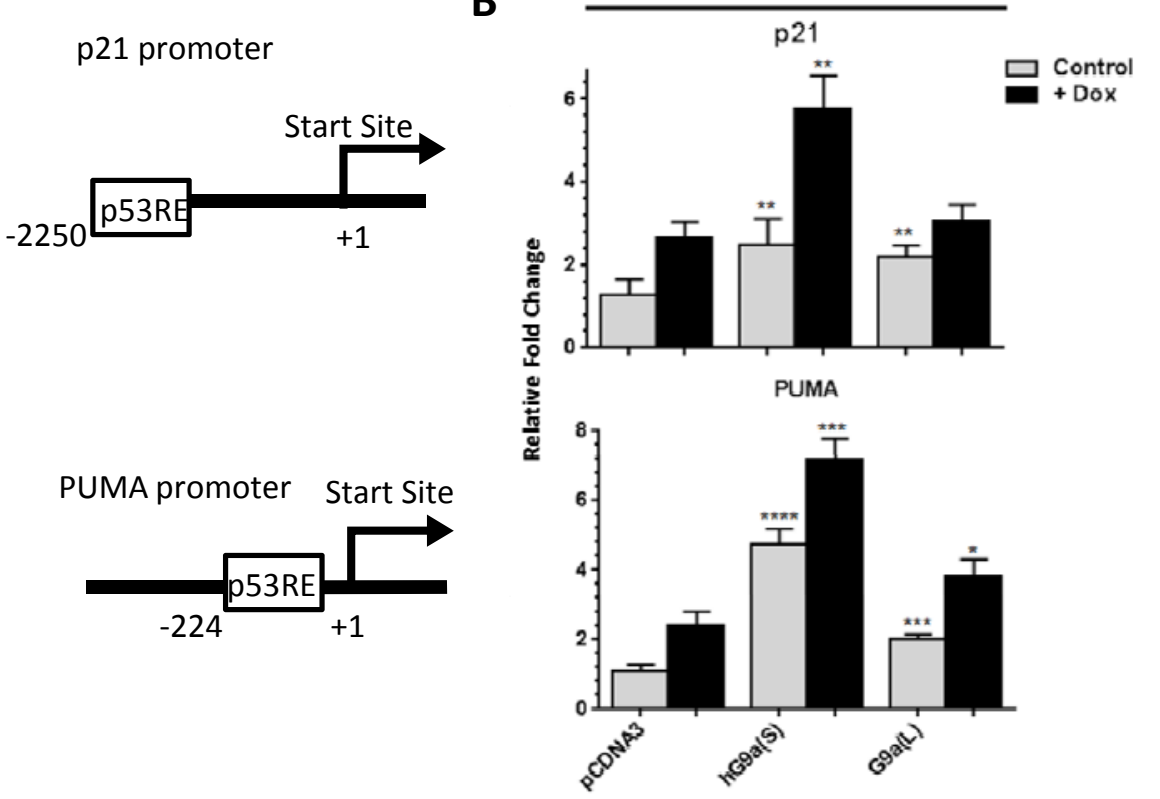

C
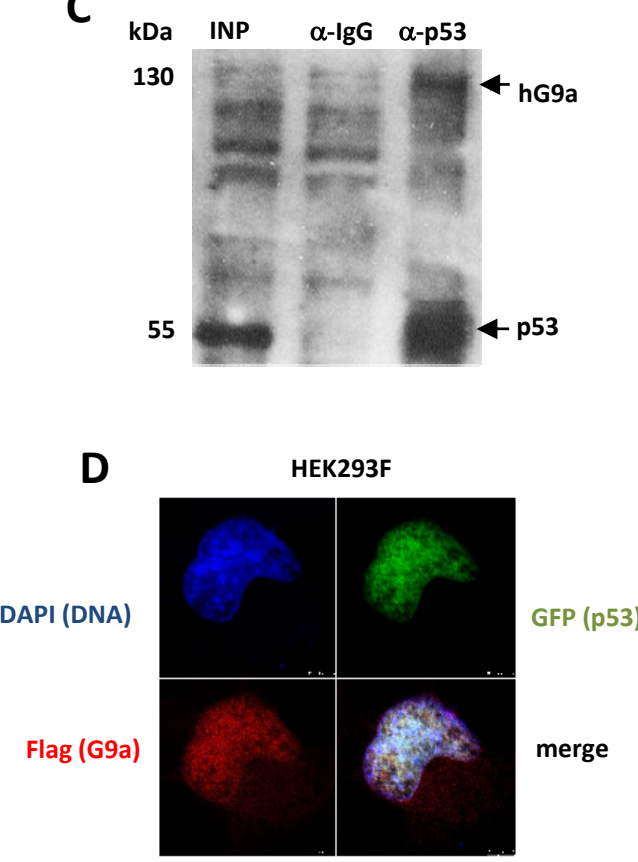

G

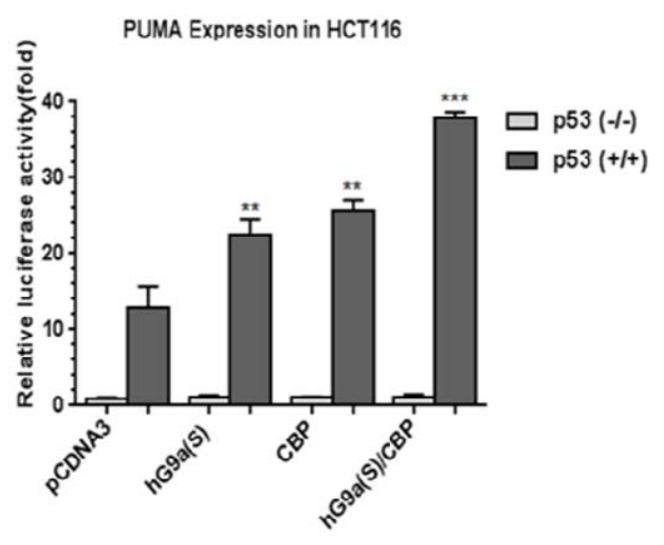

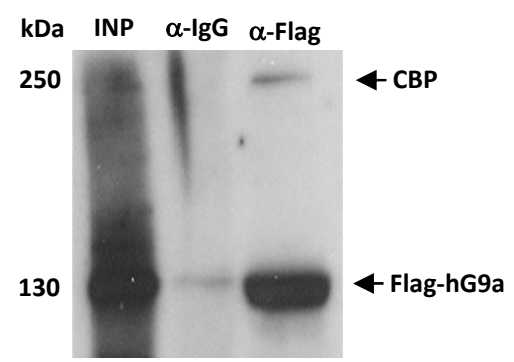

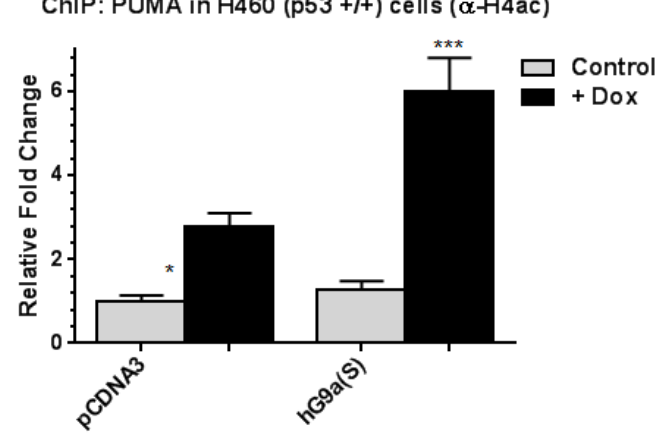

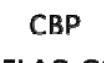

FLAG-G9

p53

$\beta$-actin 
H460 (p53 +/+) cells
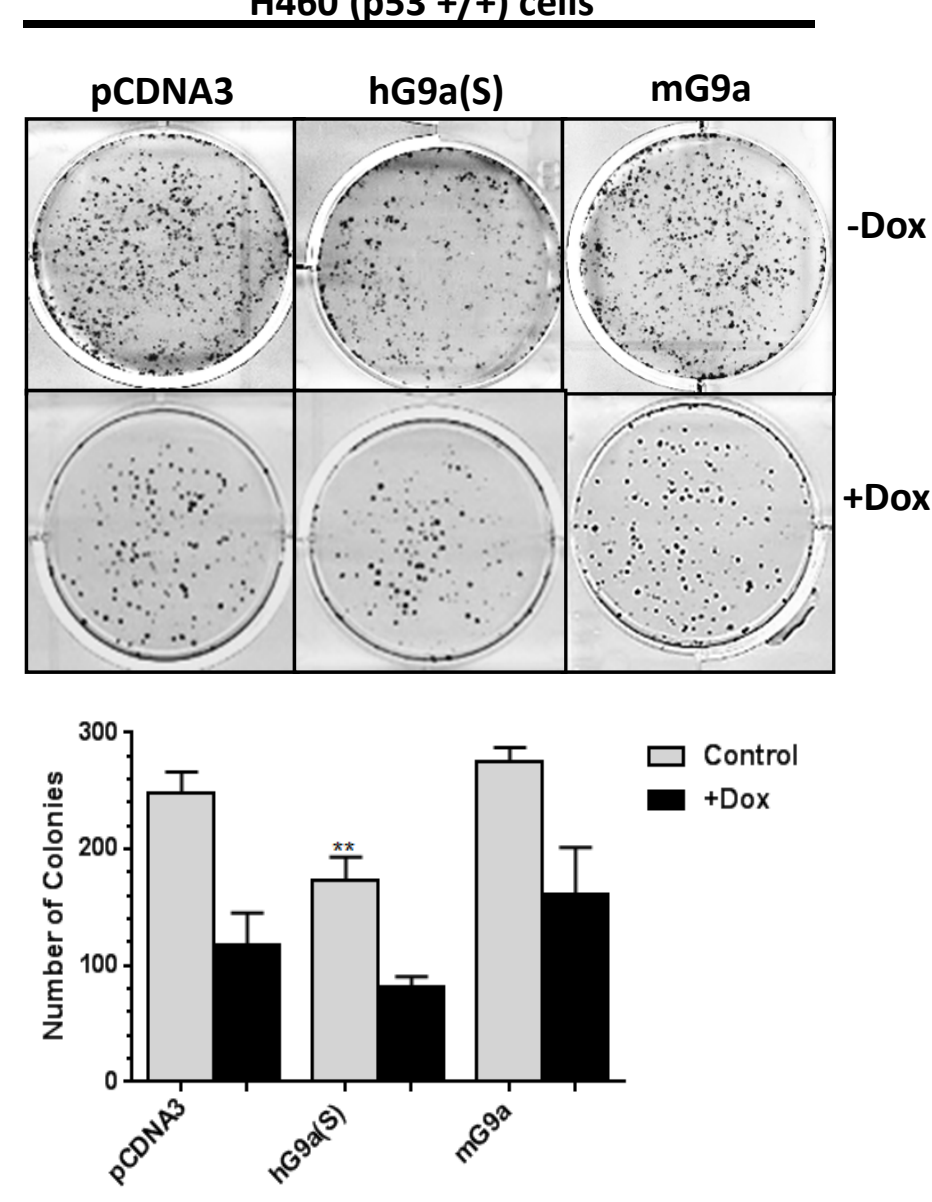

B

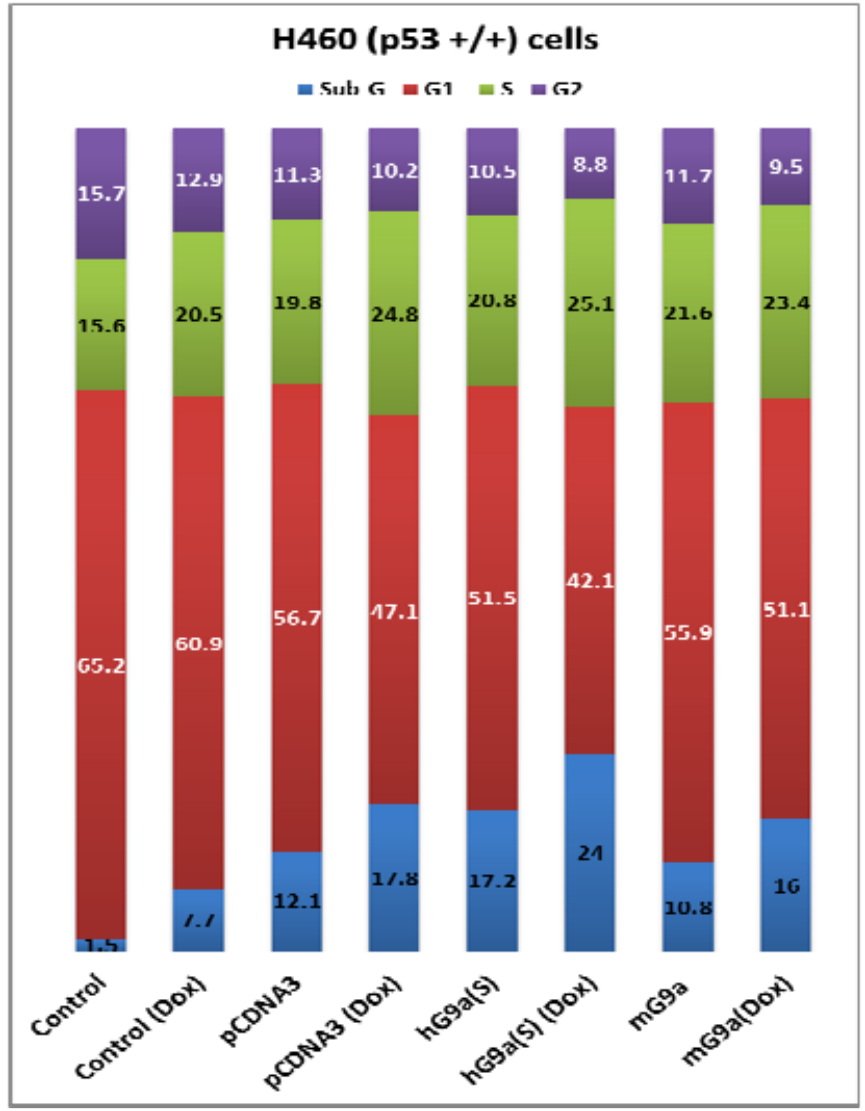

C

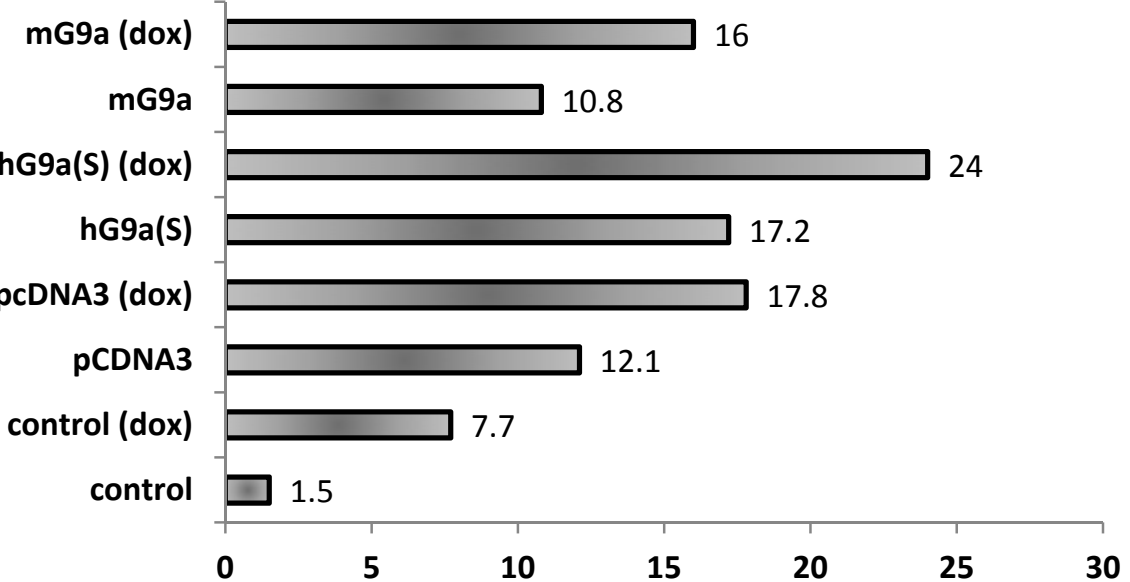

Figure 5 
GEO dataset ID: GSE31210

A GEO dataset Type: lung adenocarcinoma I-II stage

group=1: low expression of G9A

group=2: high expression of G9A

$\begin{array}{lccccc} & \mathrm{N} & \text { Observed } & \text { Expected } & (\mathrm{O}-\mathrm{E})^{\wedge} 2 / \mathrm{E} & (\mathrm{O}-\mathrm{E})^{\wedge} 2 / \\ \text { group }=1 & 96 & 18.4 & 13.5 & 1.78 & 3.34 \\ \text { group }=2 & 127 & 13.8 & 18.7 & 1.28 & 3.34\end{array}$

Chisq= 3.3 on 1 degrees of freedom, $\mathbf{p = 0 . 0 6 7 7}$

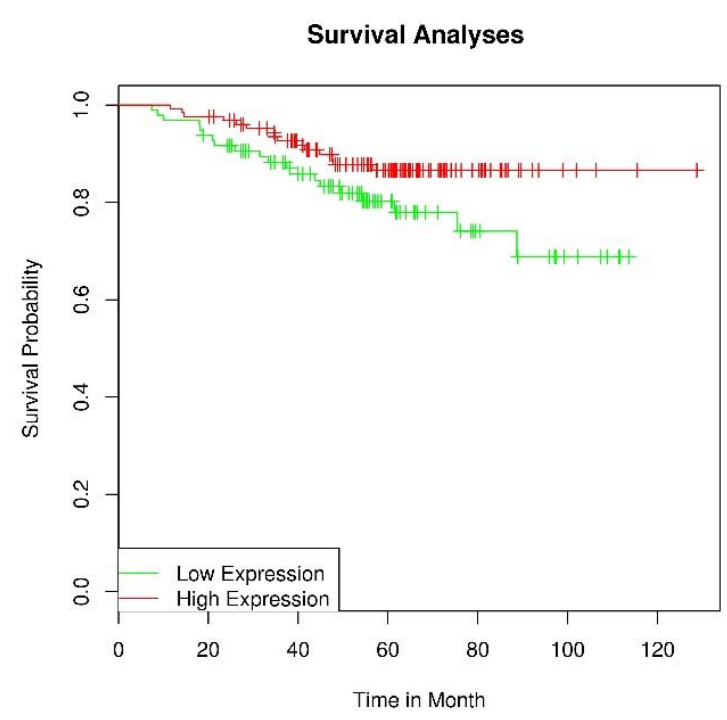

B

GEO dataset ID: GSE13213

GEO dataset Type: lung cancer
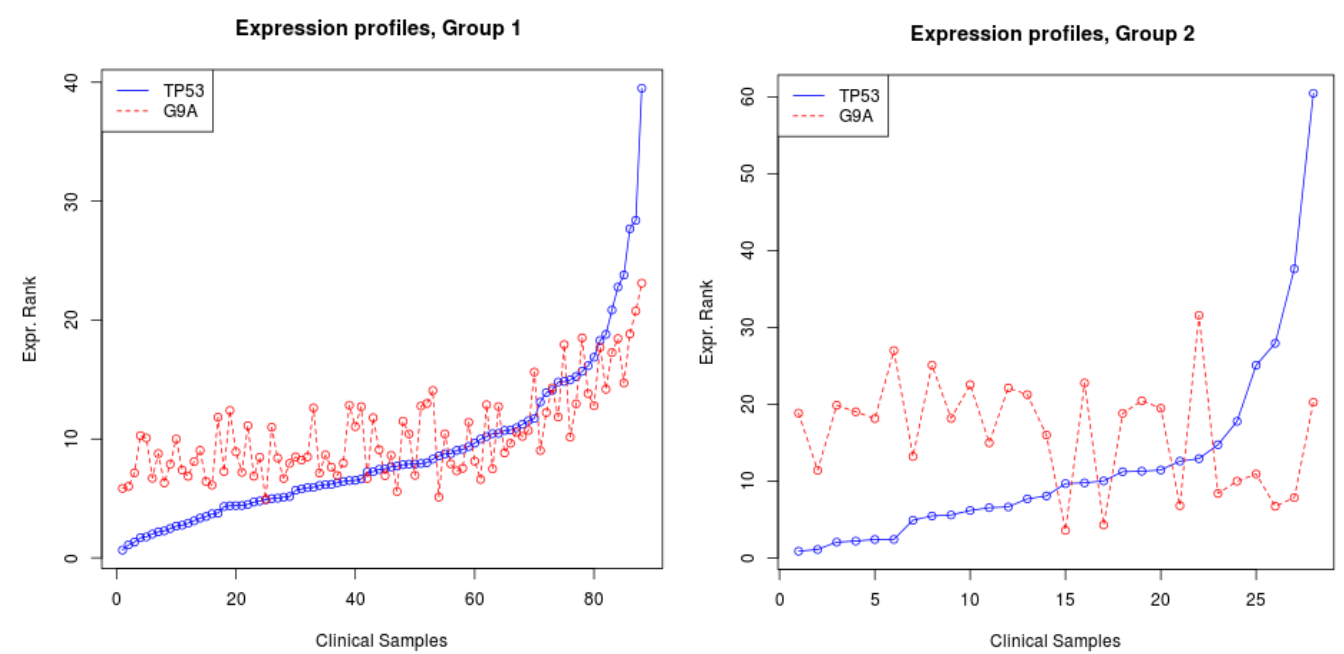

Survival differences between Group $=1$ and Group $=2$

Group=1: green , strong positive correlation (0.79) between TP53 and G9A Group=2: red , no (or negative) correlation (-0.22) between TP53 and G9A

$\begin{array}{llcccc} & \mathrm{N} & \text { Observed } & \text { Expected } & (\mathrm{O}-\mathrm{E})^{\wedge} 2 / \mathrm{E} & (\mathrm{O}-\mathrm{E})^{\wedge} 2 / \mathrm{V} \\ \text { group }=1 & 88 & 23.9 & 30.73 & 1.53 & 9.51 \\ \text { group }=2 & 28 & 14.3 & 7.48 & 6.26 & 9.51 \\ & \text { Chisq }=\text { 9.5 on 1 degrees of freedom, } \mathbf{p = 0 . 0 0 2 0 4} & \end{array}$

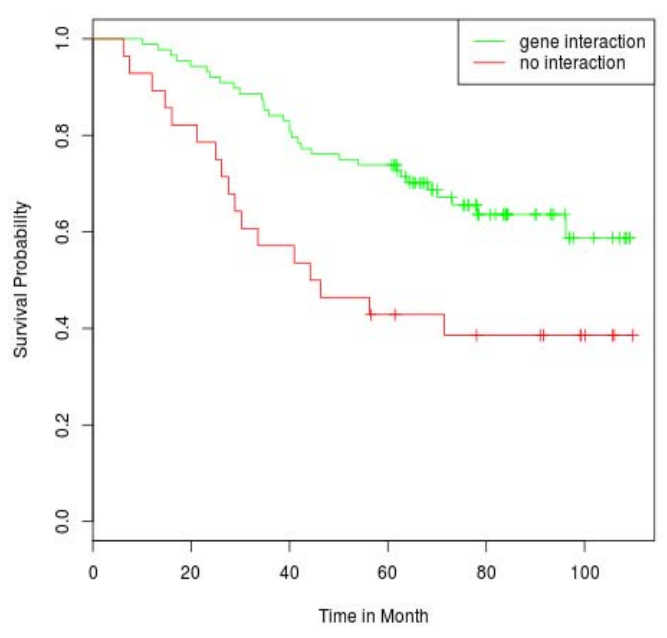

Figure 6 


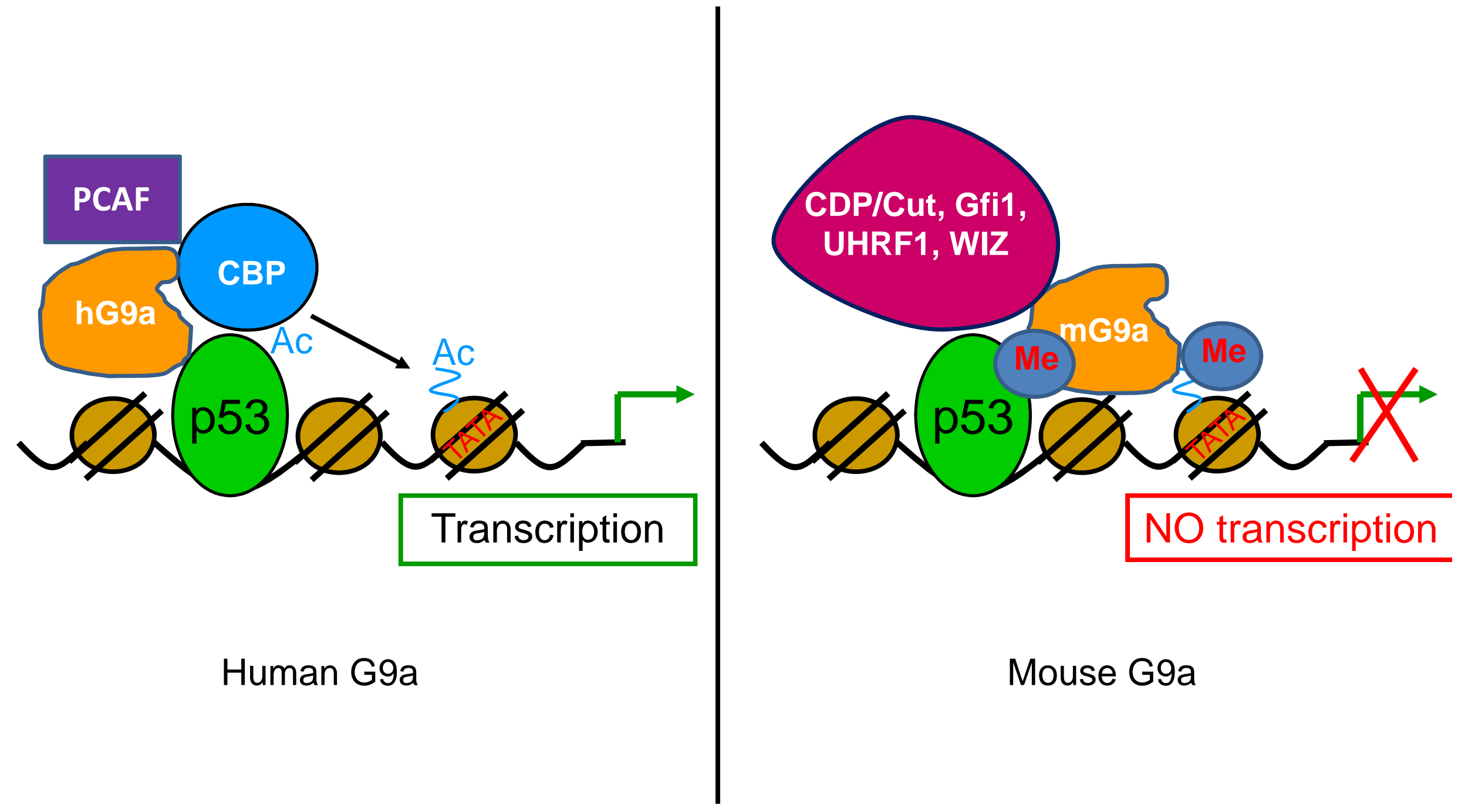

Figure 7 


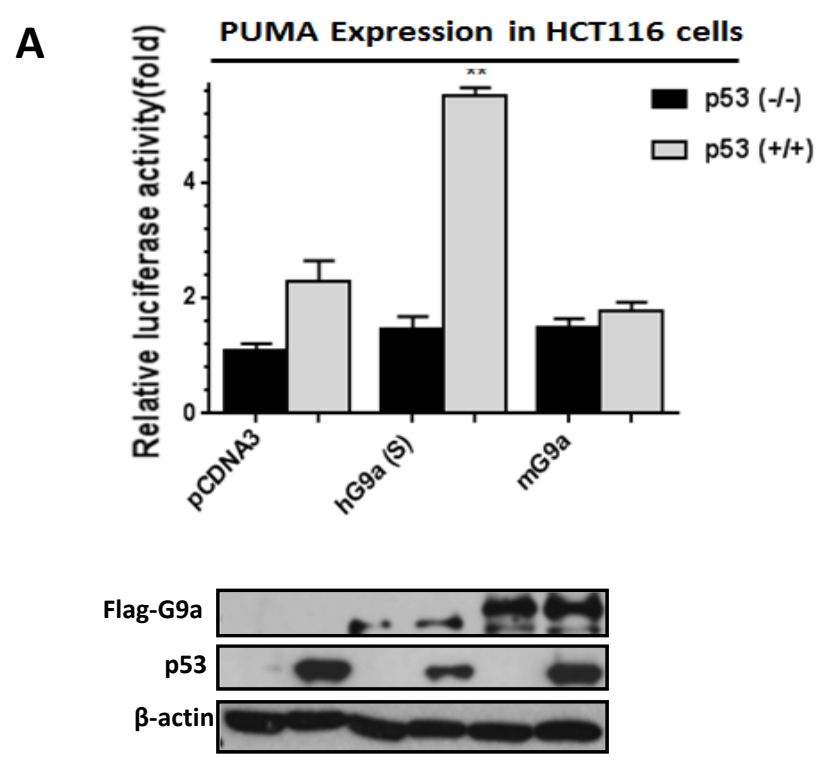

Supplementary 1 


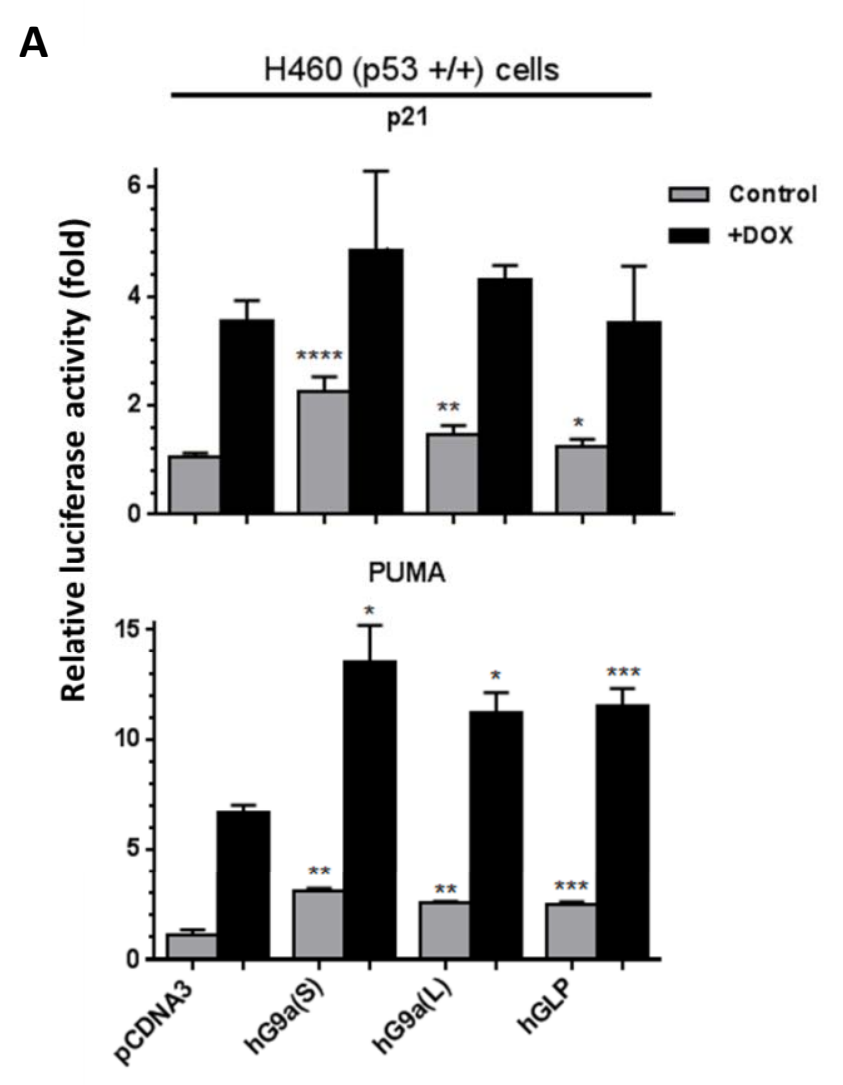

Supplementary 2 


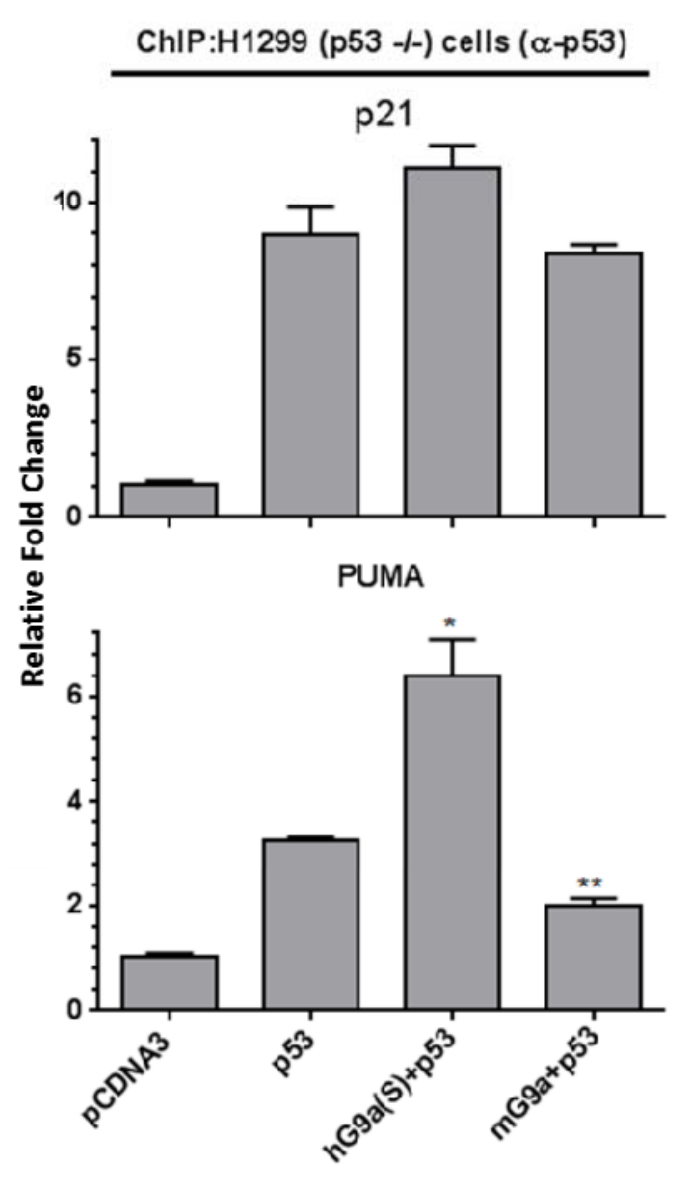

Supplementary 3 

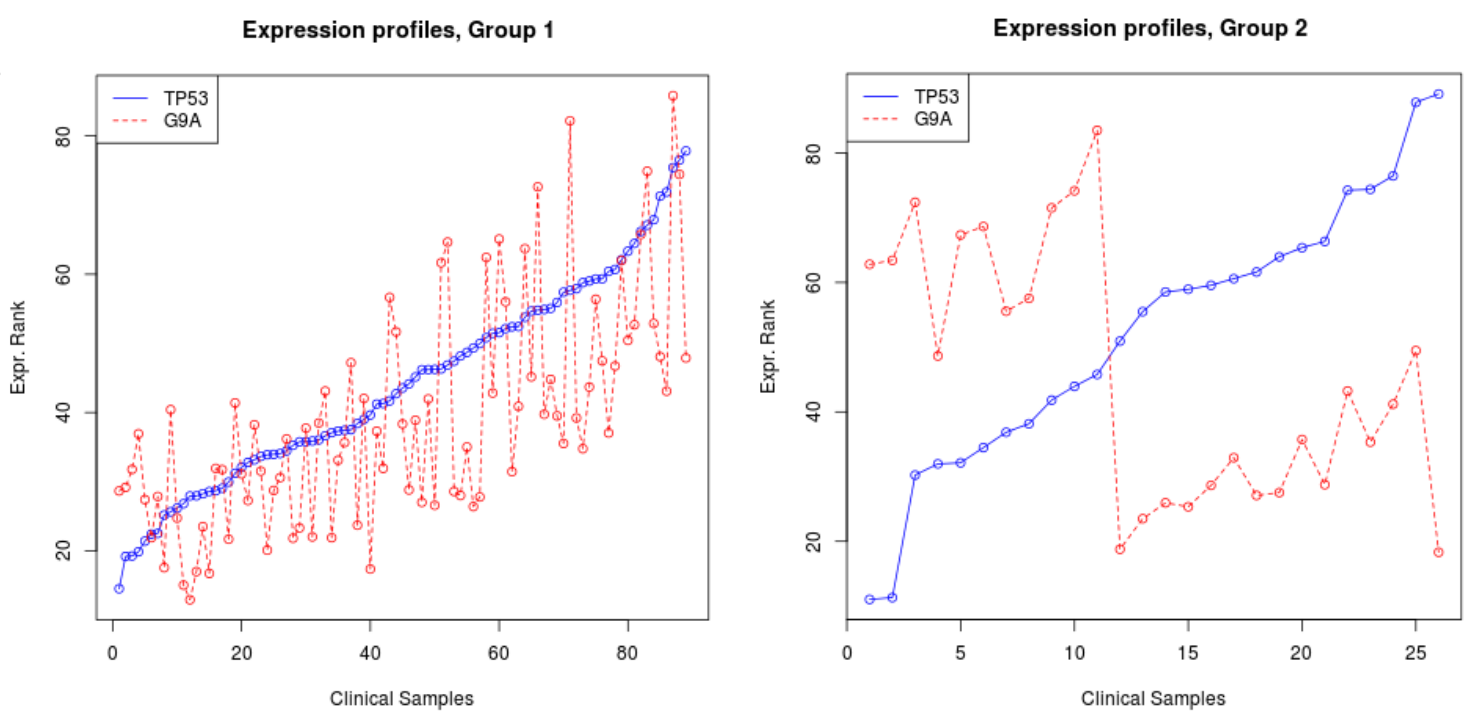

Group=1: green , strong positive correlation (0.67) between TP53 and G9A Group=2: red , no (or negative) correlation $(-0.64)$ between TP53 and G9A

\begin{tabular}{lrcccc} 
& $\mathrm{N}$ & Observed & Expected & $(\mathrm{O}-\mathrm{E})^{\wedge} 2 / \mathrm{E}$ & $(\mathrm{O}-\mathrm{E})^{\wedge} 2 / \mathrm{V}$ \\
group $=1$ & 28 & 8.17 & 10.73 & 0.611 & 6.17 \\
group $=2$ & 7 & 4.01 & 1.45 & 4.512 & 6.17 \\
& \multicolumn{4}{c}{ Chisq=6.2 } & on 1 degrees of freedom, $\mathrm{p}=0.013$
\end{tabular}

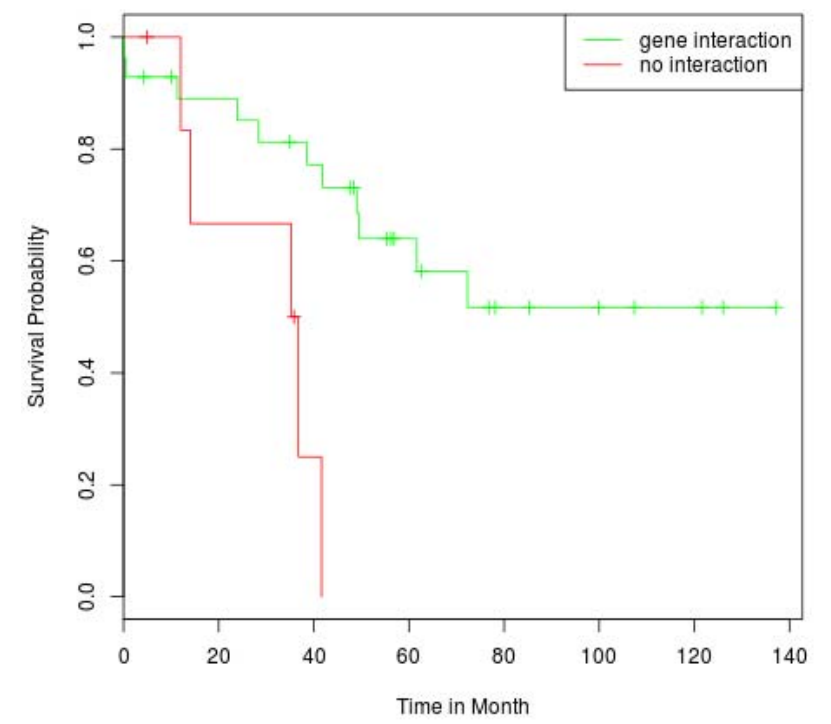

Supplementary 4 

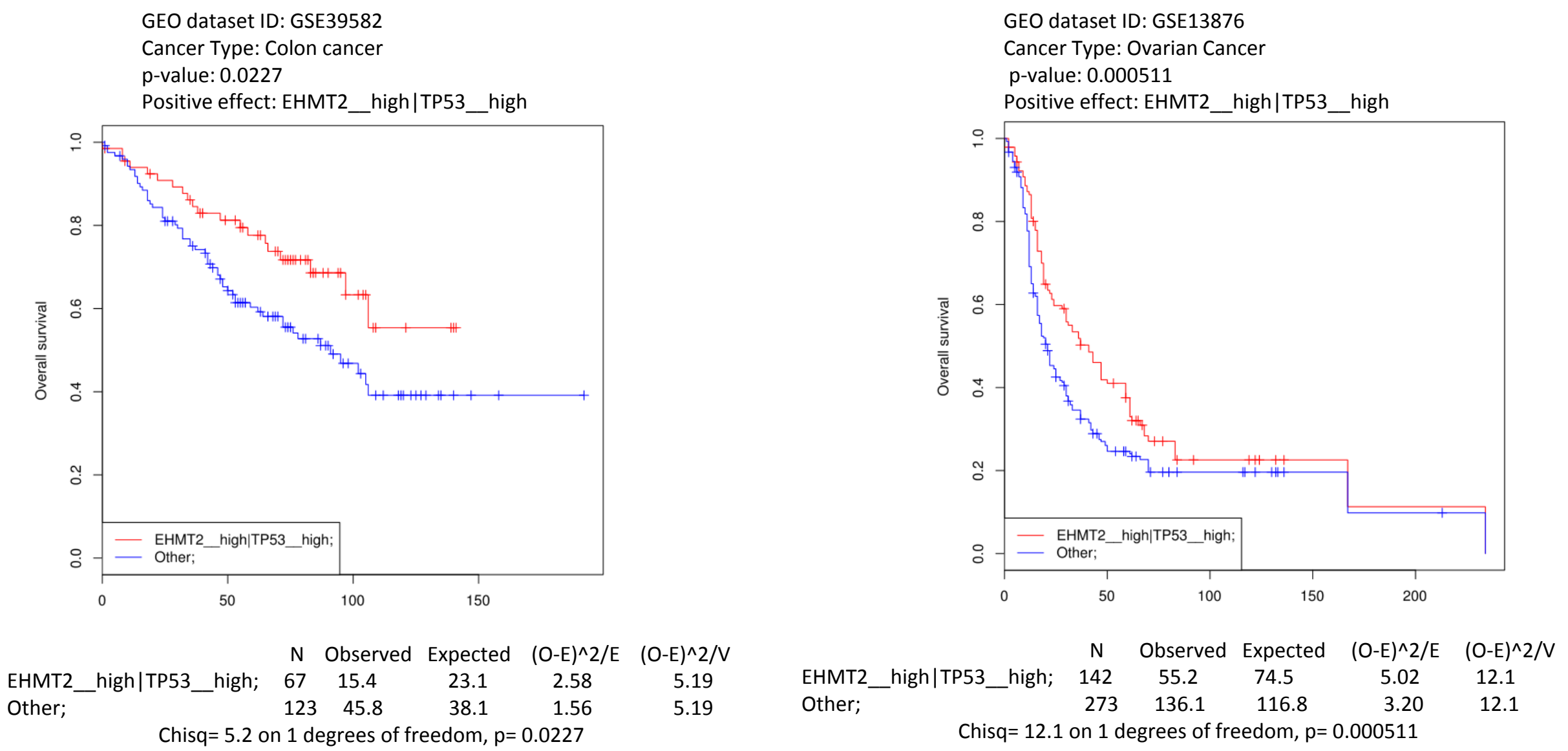

Chisq $=5.2$ on 1 degrees of freedom, $p=0.0227$ 